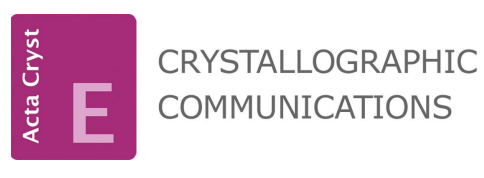

ISSN 2056-9890

Received 27 March 2020

Accepted 15 April 2020

Edited by A. J. Lough, University of Toronto, Canada

Keywords: crystal structure; hydroboration; ynamine; push-pull olefin.

CCDC reference: 1997061

Supporting information: this article has supporting information at journals.iucr.org/e

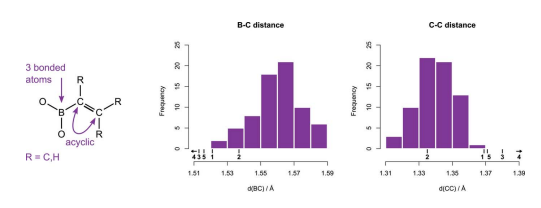

(c) (i) OPEN 2 ACCESS

\section{Structure of a push-pull olefin prepared by ynamine hydroboration with a borandiol ester}

\author{
Joël Gubler and Peter Chen*
}

\begin{abstract}
Laboratorium für Organische Chemie, ETH Zürich, Zürich, Switzerland. *Correspondence e-mail: peter.chen@org.chem.ethz.ch
\end{abstract}

$N$-[(Z)-2-(2H-1,3,2-Benzodioxaborol-2-yl)-2-phenylethenyl]- $N$-(propan-2-yl)aniline, $\mathrm{C}_{23} \mathrm{H}_{22} \mathrm{BNO}_{2}$, contains a $\mathrm{C}=\mathrm{C}$ bond that is conjugated with a donor and an acceptor group. An analysis that included similar push-pull olefins revealed that bond lengths in their $\mathrm{B}-\mathrm{C}=\mathrm{C}-\mathrm{N}$ core units correlate with the perceived acceptor and donor strength of the groups. The two phenyl groups in the molecule are rotated with respect to the plane that contains the BCCN atoms, and are close enough for significant $\pi$-stacking. Definite characterization of the title compound demonstrates, for the first time in a reliable way, that hydroboration of ynamines with borandiol esters is feasible. Compared to olefin hydroboration with borane, the ynamine substrate is activated enough to undergo reaction with the less active hydroboration reagent catecholborane.

\section{Chemical context}

Boronic esters are frequently used to transfer organic groups to transition metals, for example in the transmetallation step of the Suzuki-Miyaura reaction. Hydroboration of ynamines with borandiol esters produces amino-functionalized boronic esters in one step and perfect atom economy.<smiles>CC(C)N(/C=C(\B1Oc2ccccc2O1)c1ccccc1)c1ccccc1</smiles>

For true ynamines, to the best of our knowledge, only two attempts of such reactions have been reported so far. These either failed (Witulski et al., 2000) or were reported without reaction details and characterization data (Zhuo et al., 2001). More recently it was found that the exceptionally active Pier's borane, $\mathrm{HB}\left(\mathrm{C}_{6} \mathrm{~F}_{5}\right)_{2}$, can readily hydroborate 1-propynyl2,2,6,6-tetramethylpiperidine (Wang et al., 2018). Borandiol esters are expected to be less reactive because the electron deficiency at the boron is reduced by $\pi$-donation from the oxygen atoms.

Given the limited precedent for ynamine hydroboration, the more comprehensive literature for enamine hydroboration was consulted (Goralski \& Singaram, 2012; Dembitsky et al., 2002), as their reactivity is expected to be controlled by similar effects. Compared to simple olefin substrates, conjugation of the $\mathrm{C}=\mathrm{C}$ bond with nitrogen dictates the regioselectivity and increases the reactivity of enamines. However, the presence of a nitrogen atom in the reactant and product enables the formation of unreactive Lewis acid-base adducts with the 


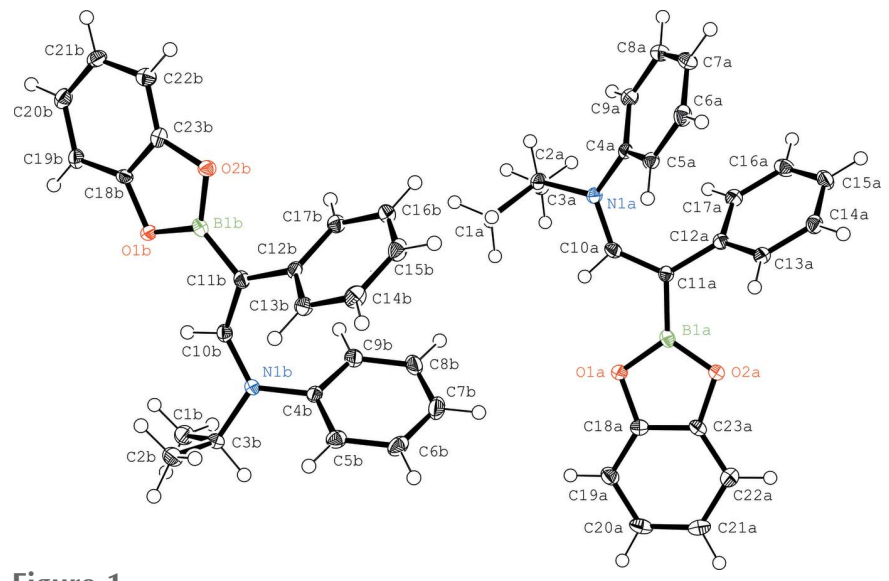

Figure 1

The molecular structures of the two independent molecules of the title compound $\mathbf{1}$ with displacement ellipsoids drawn at the $50 \%$ probability level.

hydroborating reagent. Building on the vast knowledge of the reactivity of different borane-amine adducts in hydroboration (Brown \& Murray, 1984; Brown et al., 1999), a bulky iso-propyl and an phenyl group were selected as substituents for the ynamine nitrogen. The former should weaken adducts for steric reasons, whereas the phenyl group is expected to reduce the nucleophilicity of the nitrogen by conjugation.

Ynamine hydroboration using a borandiol ester was reinvestigated and succeeded for a substrate that follows the developed design principles. The product of such a reaction contains a $\mathrm{C}=\mathrm{C}$ double bond flanked by both an electrondonating group (EDG), the amine, and an electron-withdrawing group (EWG), the boronate. Therefore it belongs to the class of push-pull (captodative) olefins, which are known to have unusual properties such as weak $\pi$-bonds or biradical reactivity (Viehe et al., 1985).

\section{Structural commentary}

The asymmetric unit (Fig. 1) contains two almost identical (r.m.d.s $=0.11 \AA$ ) independent molecules. As judged by the $\mathrm{B} 1-\mathrm{C} 11-\mathrm{C} 10-\mathrm{N} 1$ torsion angles of 171.6 (2) and $175.5(2)^{\circ}$, the central $\mathrm{C}-\mathrm{C}$ bond is only slightly twisted from planarity. The two phenyl groups in the molecule are rotated, by 43 and $49^{\circ}$, with respect to that plane. The centroids of two phenyl groups in one molecule are on average $3.747 \AA$ apart, which suggests intramolecular $\pi$-stacking. The mean distances are $1.521 \AA$ for the $\mathrm{B} 1-\mathrm{C} 11$ bond, $1.365 \AA$ for the $\mathrm{C} 10-\mathrm{N} 1$ bond and $1.369 \AA$ for the central C10-C11 bond.

\section{Supramolecular features}

There is a short intermolecular contact between the boron atom and an aniline ortho- $\mathrm{H} B 1 B \cdots \mathrm{H} 9 A\left(1-x, 1-y, \frac{1}{2}+z\right)=$ $2.771 \AA$ and $\mathrm{B} 1 A \cdots \mathrm{H} 5 B\left(1-x,-y,-\frac{1}{2}+z\right)=2.856 \AA$ $\left(\Sigma \mathrm{r}_{\mathrm{vdW}}[\mathrm{B}, \mathrm{H}]=3.11 \AA\right)$. The shortest intermolecular distances between the catechol unit and boron are $\mathrm{B} 1 A \cdots \mathrm{C} 21 B^{\mathrm{i}}=$ 3.540 (4) $\AA, \quad \mathrm{B} 1 A \cdots \mathrm{C} 20 B^{\mathrm{i}}=3.686(4) \AA\left(\Sigma \mathrm{r}_{\mathrm{vdw}}[\mathrm{B}, \mathrm{C}]=\right.$
Table 1

Comparison of bond lengths (in $\AA$ ) in $\mathbf{1}$ with those in the similar compounds 2-5.

Average distances and standard deviations are given whenever there is more than one molecule in the asymmetric unit. Typical bond lengths for vinylboranes and conjugated enamines were obtained from statistical analysis.

\begin{tabular}{lllll}
\hline Compound & $\mathrm{B}-\mathrm{C}$ & $\mathrm{C}-\mathrm{C}$ & $\mathrm{C}-\mathrm{N}$ & $\mathrm{CCDC}$ \\
\hline $\mathbf{1}$ & $1.521(3)$ & $1.369(3)$ & $1.365(3)$ & title compound \\
$\mathbf{2}$ & $1.537(4)$ & $1.335(4)$ & $1.394(3)$ & $861787^{a}$ \\
$\mathbf{3}$ & $1.513(4)$ & $1.380(3)$ & $1.362(3)$ & 1529736 \\
$\mathbf{4}$ & $1.491(7)$ & $1.393(6)$ & $1.341(6)$ & $1843575^{c}$ \\
$\mathbf{5}$ & $1.516(2)$ & $1.371(2)$ & $1.350(2)$ & $1997665^{d}$ \\
Vinyl boronates & $1.561(15)$ & $1.341(12)$ & - & - \\
Enamines & - & $\sim 1.34$ & $\sim 1.36$ & - \\
\hline
\end{tabular}

Notes: (a) Hatayama \& Okuno (2012); (b) Liu et al. (2017); (c) Wang et al. (2018); (d) new.

$3.68 \AA), \mathrm{B} 1 A \cdots \mathrm{H} 21 B^{\mathrm{i}}=3.113 \AA$ and $\mathrm{B} 1 A \cdots \mathrm{H} 20 B^{\mathrm{i}}=3.381 \AA$ [symmetry code: (i) $\frac{1}{2}+x, 1-y, z$ ]. In addition there is a short contact between one of the other catechol hydrogen atoms and the meta-carbon of the aniline, $\mathrm{H} 22 B \cdots \mathrm{C} 6 B(x, 1+y, z)$ of $2.877 \AA\left(\Sigma \mathrm{r}_{\mathrm{vdW}}[\mathrm{C}, \mathrm{H}]=2.97 \AA\right)$. All of these interactions involve atoms that are part of arenes and could be seen as intermolecular $\pi$-stacking.

Methyl hydrogen atoms of the isopropyl group are at van der Waals distances with one of the oxygen atoms $\left[\mathrm{O} 2 A \cdots \mathrm{H} 2 B A\left(1-x,-y,-\frac{1}{2}+z\right)=2.698 \AA\right.$, O $2 B \cdots$ $\left.\mathrm{H} 3 A B\left(1-x, 1-y, \frac{1}{2}+z\right)=2.631 \AA, \Sigma \mathrm{r}_{\mathrm{vdw}}[\mathrm{O}, \mathrm{H}]=2.70 \AA\right]$ and with one of the anilic meta-H atoms [H8A $\cdots \mathrm{H} 2 B C(1-x$, $\left.\left.-y,-\frac{1}{2}+z\right)=2.388 \AA, \Sigma \mathrm{r}_{\mathrm{vdw}}[\mathrm{H}, \mathrm{H}]=2.40 \AA\right]$. The nitrogen atom is sterically shielded by surrounding groups and does not have any close intermolecular neighbours.

\section{Database survey}

Contributions from the zwitterionic resonance structure ${ }^{-} \mathrm{B}=\mathrm{C}-\mathrm{C}=\mathrm{N}^{+}$are expected to increase with donor and acceptor group strength. This should be observable as a shortening of the $\mathrm{B}-\mathrm{C}$ and $\mathrm{C}-\mathrm{N}$ bonds and an elongation of the $\mathrm{C}-\mathrm{C}$ bond. Following this idea, bond lengths of $\mathbf{1}$ were compared to those in the structurally related compounds $\mathbf{2}-\mathbf{5}$
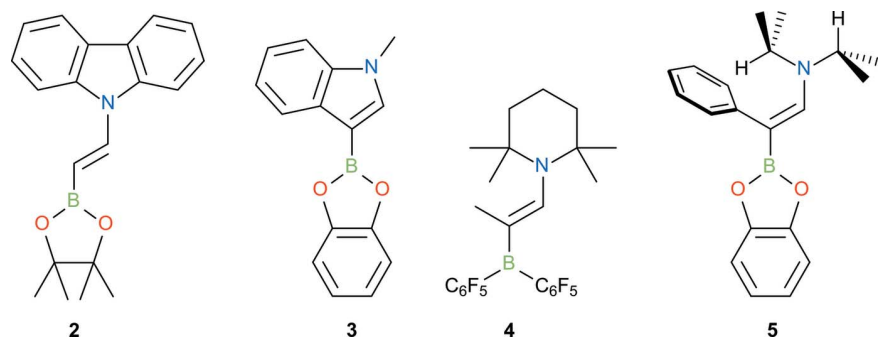

Figure 2

Chemical structure of reference compounds. 9- $[(E)-2-(4,4,5,5-$ Tetramethyl-1,3,2-dioxaborolan-2-yl)ethenyl]-9H-carbazole, 2 (Hatayama \& Okuno, 2012), 3-(2H-1,3,2-benzodioxaborol-2-yl)-1-methyl-1 $H$-indole, 3 (Liu et al., 2017), 1-\{(Z)-2-[bis(pentafluorophenyl)boranyl]prop-1-en-1yl $-2,2,6,6$-tetramethylpiperidine, 4 (Wang et al., 2018) and $N-[(Z)-2-(2 H$ 1,3,2-benzodioxaborol-2-yl)-2-phenylethenyl]- $N$-(propan-2-yl)propan-2amine, 5 (CCDC 1997665). 


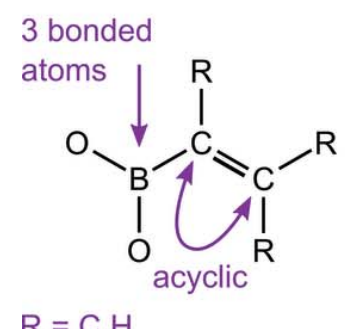

$\mathrm{R}=\mathrm{C}, \mathrm{H}$
B-C distance

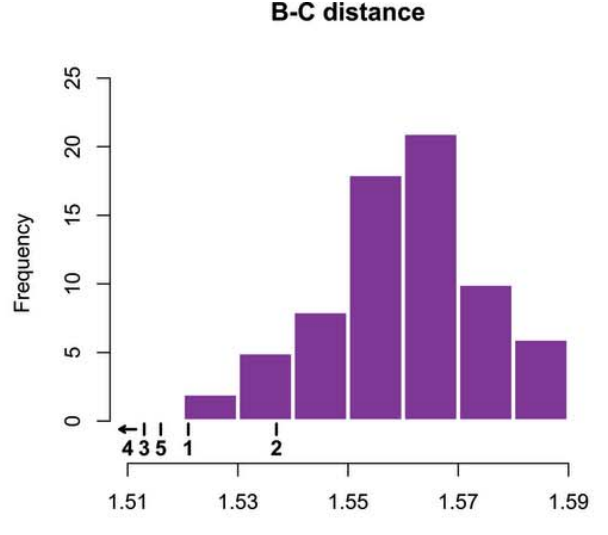

$\mathrm{d}(\mathrm{BC}) / \AA$
C-C distance

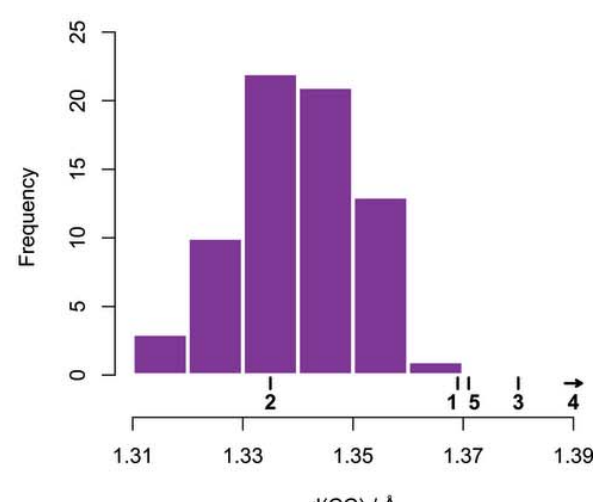

$\mathrm{d}(\mathrm{CC}) / \AA$

Figure 3

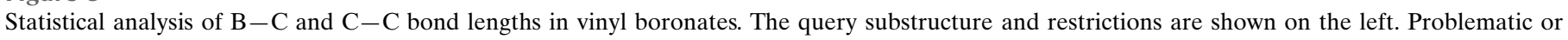
irrelevant structures were removed. The bond distances of reference compounds are marked.

(Table 1, Fig. 2). $\mathrm{C}-\mathrm{N}$ bond lengths are $1.341 \AA$ (4), $1.350 \AA$ (5), $1.362 \AA$ (3), $1.365 \AA$ (1), $1.394 \AA$ (2). These numbers follow the expected $\mathrm{N}$-donor strength, when the latter is estimated by the number of conjugating substituents on the nitrogen: piperidine, diisopropyl $>$ aniline $>$ indole, carbazole. B - C lengths are $1.491 \AA$ (4), $1.513 \AA$ (3), $1.516 \AA$ (5), $1.521 \AA$ (1), $1.537 \AA$ (2). Similarly, these numbers follow the Bacceptor strength: $\mathrm{B}\left(\mathrm{C}_{6} \mathrm{~F}_{5}\right)_{2}>$ catecholboryl $>$ pinacolboryl (Adamczyk-Woźniak et al., 2011). Following this, the zwitterionic resonance structure is most important in $\mathbf{4}$, which has a strong donor and a strong acceptor. On the other end of the scale lies 2, which has a weak donor and a weak acceptor. The other molecules, including $\mathbf{1}$, lie between these two extremes.

In order to compare with olefins that either have a donor or an acceptor group, the Cambridge Structural Database (CSD, Version 5.41, update of November 2019; Groom et al., 2016) was searched for vinyl boronates and enamines. Bond-length distributions and the exact query structures are shown in Fig. 3 and Fig. 4. The data set for vinyl boronates consists of about $90 \%$ of pinacol boronates and contains only a few catechol boronates. Compared with typical bond lengths in this data set, the $\mathrm{B}-\mathrm{C}$ bond is shorter and the $\mathrm{C}=\mathrm{C}$ bond is longer in
1-5, which indicates stronger conjugation. The only exception is 2 , whose $\mathrm{C}=\mathrm{C}$ bond is shorter.

For enamines the $\mathrm{C}-\mathrm{C}$ bond length has an average of $1.341 \AA$, which is about $0.025 \AA$ longer than the value of 1.316 (15) $\AA$ for regular internal olefins (Allen et al., 2006). In $\mathbf{1}, \mathbf{3}, \mathbf{4}$ and $\mathbf{5}$, the $\mathrm{C}-\mathrm{C}$ bonds are on average $1.378 \AA$, and thereby longer than in enamines. $\mathrm{C}-\mathrm{N}$ bond lengths for enamines are distributed more uniformly than $\mathrm{C}-\mathrm{C}$ lengths. Inspection of the structures in which $\mathrm{C}-\mathrm{N}$ distances are longer than $1.39 \AA$ revealed that these structures typically either have a nitrogen whose lone pair is not coplanar with the $\mathrm{C}=\mathrm{C}$ bond, or a nitrogen that is part of a carbazole or morpholine. As conjugation with the formal double bond between $\mathrm{C} 10$ and $\mathrm{C} 11$ is absent or reduced in these, only structures with $\mathrm{C}-\mathrm{N}$ bond lengths below $1.39 \AA$ A were used for comparison. The average $\mathrm{C}-\mathrm{N}$ bond length of about $1.36 \AA$ for that subset is similar to the $\mathrm{C}-\mathrm{N}$ bond lengths in $\mathbf{1}, \mathbf{3}, \mathbf{4}$ and 5. Overall, comparison with enamines reveals that $\mathrm{C}-\mathrm{C}$ bonds are longer in push-pull olefins, whereas $\mathrm{C}-\mathrm{N}$ bond lengths are unaffected. This suggests that conjugation with the boron affects the $\mathrm{C}-\mathrm{C}$ bond length more than the $\mathrm{C}-\mathrm{N}$ bond length.

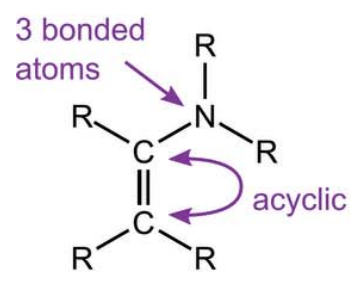

$\mathrm{R}=\mathrm{C}^{*}, \mathrm{H}$

$C^{*}$ : carbon atom that is not bound to any atom by a double or triple bond

Figure 4

Statistical analysis of $\mathrm{C}-\mathrm{C}$ and $\mathrm{C}-\mathrm{N}$ bond lengths in enamines. The query substructure and restrictions are shown on the left. Problematic or irrelevant structures were removed. The bond distances of reference compounds are marked.
C-C distance

C-N distance
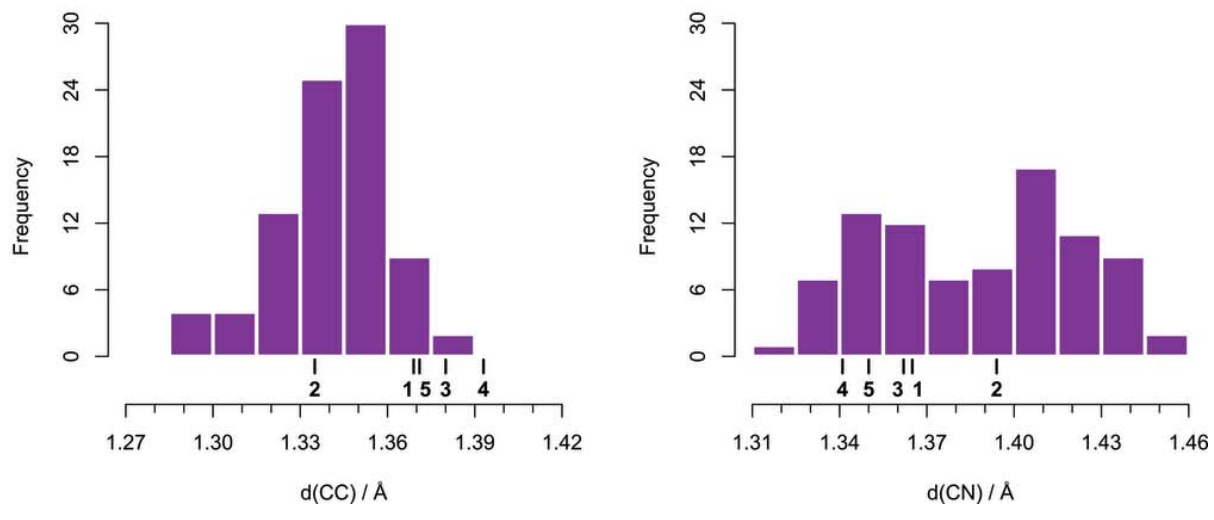


\section{Synthesis and crystallization}

The title compound was prepared by the multi-step sequence shown in Fig. 5.

$\boldsymbol{N}$-isopropyl- $\boldsymbol{N}$-(phenylethynyl)aniline: In a $100 \mathrm{ml}$ Schlenk flask, $5.8 \mathrm{ml}$ of $N$-isopropyl amine ( $40 \mathrm{mmol}, 1.0$ eq.) were diluted with $40 \mathrm{ml}$ of dry THF. $24.7 \mathrm{ml}$ of a $n$-BuLi solution in hexanes (1.62 mol l-1, $40 \mathrm{mmol}, 1.0$ eq.) were added over $5 \mathrm{~min}$ at $195 \mathrm{~K}$. A colourless solid formed and after $15 \mathrm{~min}$ the suspension was warmed to room temperature over $30 \mathrm{~min}$. Upon addition of $5.69 \mathrm{~g}$ of 2-chloroethynylbenzene (96\%, $40 \mathrm{mmol}, 1.0$ eq., prepared according to $\mathrm{Li}$ et al., 2014), the reaction mixture turned black. The sealed Schlenk flask was heated in an oil bath at $338 \mathrm{~K}$ (caution: the closed flask may burst if this temperature is exceeded). The reaction progress was monitored by GC-FID. After $6 \mathrm{~h}$ the reaction mixture was cooled to room temperature. $100 \mathrm{ml}$ of tert-butyl methyl ether were added, the organic phase washed with ice-cold water (3 $\times 50 \mathrm{ml}$ ), dried with $\mathrm{MgSO}_{4}$ and concentrated on the rotavap. $8.25 \mathrm{~g}$ of black viscous liquid were obtained and purified by Kugelrohr distillation $(433 \mathrm{~K}, 0.2 \mathrm{mbar})$ to yield $6.34 \mathrm{~g}$ (purity $83 \mathrm{wt} \%$, yield $56 \%$ ) of the colourless liquid $N$-isopropyl- $N$ (phenylethynyl)aniline. ${ }^{1} \mathrm{H} \mathrm{NMR}\left(400 \mathrm{MHz}, \mathrm{CDCl}_{3}\right): \delta(\mathrm{ppm})$ $=1.40\left(d, 6.5 \mathrm{~Hz}, 6 \mathrm{H}, 2 \times \mathrm{CH}_{3}\right), 4.10$ (hept, $6.4 \mathrm{~Hz}, 1 \mathrm{H}, \mathrm{CH}$ of $i$-Pr), $6.92(t t, 7.4 \mathrm{~Hz}, 1.2 \mathrm{~Hz}, 1 \mathrm{H}$, para-H of aniline), 7.16-7.33 ( $m, 7 \mathrm{H}$, arene $\mathrm{H}), 7.38-7.41$ ( $m, 2 \mathrm{H}$, ortho-H of phenyl group). ${ }^{13} \mathrm{C}\left\{{ }^{1} \mathrm{H}\right\} \mathrm{NMR}\left(100 \mathrm{MHz}, \mathrm{CDCl}_{3}\right): \delta(\mathrm{ppm})=20.6\left(s, 2 \times \mathrm{CH}_{3}\right)$, $49.6(s, \mathrm{CH}$ of $i$-Pr), 72.0 ( $s$, alkynic carbon farther from $\mathrm{N}$ ), 86.4 ( $s$, alkynic carbon closer to $\mathrm{N}$ ), 115.5 ( $s$, ortho-C of aniline), 120.8 ( $s$, para-C of aniline), 125.2 ( $s$, ipso-C of phenyl group), 126.2 ( $s$, para-C of phenyl group), 128.4 ( $s$, meta-C of phenyl group), 129.3 ( $s$, meta-C of aniline), 130.2 ( $s$, ortho-C of phenyl group), 144.4 (s, ipso-C of aniline). EI-MS $(70 \mathrm{eV}) \mathrm{m} / z$ = 236, $235\left(M^{+}\right), 220\left(M^{+}-\mathrm{CH}_{3}\right), 194,193,192\left(M^{+}-\mathrm{C}_{3} \mathrm{H}_{7}\right)$, $165,117,115,90,89,77,63,51,43$. ATR-IR $v\left(\mathrm{~cm}^{-1}\right)(\% \mathrm{~T})=$ $534(70), 629(61), 688(22), 745(18), 783(82), 865(87)$, 881 (87), 904 (87), 996 (80), 1025 (73), 1056 (66), 1129 (70), 1147 (52), 1170 (67), 1254 (41), 1312 (67), 1348 (77), 1367 (76), 1396 (51), 1490 (38), 1594 (46), 1932 (96), 2217 (38, C $\equiv \mathrm{C}-$ stretch), 2872 (94), 2932 (91), 2976 (80), 3034 (93), 3057 (93).

$\boldsymbol{N}$-[(Z)-2-(2H-1,3,2-benzodioxaborol-2-yl)-2-phenylethenyl]-N-(propan-2-yl)aniline: Under a counterflow of argon, $3.08 \mathrm{~g}$ of $N$-isopropyl- $N$-(phenylethynyl)aniline (13.1 mmol, 1.0 eq.) were placed in an oven-dried $20 \mathrm{ml}$ Schlenk flask with a Young valve. The flask and its contents were purged three times by applying high vacuum followed by flushing with argon. $6.5 \mathrm{ml}$ dry tert-butyl methyl ether were added and the mixture stirred vigorously to ensure mixing of the two liquids. $2.1 \mathrm{ml}$ of catecholborane (19.5 mmol, $1.5 \mathrm{eq}$.) were added, the flask closed, and the reaction mixture heated to $323 \mathrm{~K}$ for $16 \mathrm{~h}$. Cooling to room temperature led to the precipitation of the

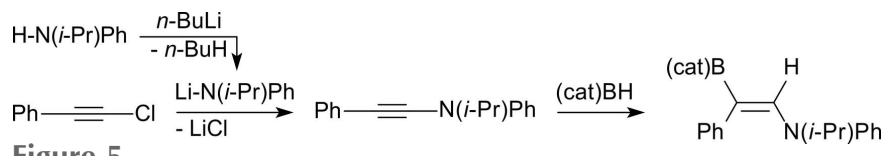

Figure 5

Reaction sequence used for the synthesis of the title compound.
Table 2

Experimental details.

\begin{tabular}{|c|c|}
\hline \multicolumn{2}{|l|}{ Crystal data } \\
\hline Chemical formula & $\mathrm{C}_{23} \mathrm{H}_{22} \mathrm{BNO}_{2}$ \\
\hline$M_{\mathrm{r}}$ & 355.22 \\
\hline Crystal system, space group & Orthorhombic, $P c a 2_{1}$ \\
\hline Temperature (K) & 100 \\
\hline$a, b, c(\AA)$ & $\begin{array}{l}17.8540(11), 11.5361(6) \\
\quad 18.5366(12)\end{array}$ \\
\hline$V\left(\AA^{3}\right)$ & $3817.9(4)$ \\
\hline$Z$ & 8 \\
\hline Radiation type & Мо $K \alpha$ \\
\hline$\mu\left(\mathrm{mm}^{-1}\right)$ & 0.08 \\
\hline Crystal size $(\mathrm{mm})$ & $0.38 \times 0.2 \times 0.07$ \\
\hline \multicolumn{2}{|l|}{ Data collection } \\
\hline Diffractometer & Bruker-Nonius Kappa APEXII \\
\hline Absorption correction & $\begin{array}{l}\text { Multi-scan ( } S A D A B S ; \text { Bruker, } \\
\text { 2015) }\end{array}$ \\
\hline$T_{\min }, T_{\max }$ & $0.703,0.733$ \\
\hline $\begin{array}{l}\text { No. of measured, independent and } \\
\text { observed }[I>2 \sigma(I)] \text { reflections }\end{array}$ & $60829,8781,7379$ \\
\hline$R_{\text {int }}$ & 0.047 \\
\hline$(\sin \theta / \lambda)_{\max }\left(\AA^{-1}\right)$ & 0.652 \\
\hline \multicolumn{2}{|l|}{ Refinement } \\
\hline$R\left[F^{2}>2 \sigma\left(F^{2}\right)\right], w R\left(F^{2}\right), S$ & $0.039,0.086,1.03$ \\
\hline No. of reflections & 8781 \\
\hline No. of parameters & 491 \\
\hline No. of restraints & 1 \\
\hline $\mathrm{H}$-atom treatment & $\mathrm{H}$-atom parameters constrained \\
\hline$\Delta \rho_{\max }, \Delta \rho_{\min }\left(\mathrm{e} \AA^{-3}\right)$ & $0.25,-0.24$ \\
\hline
\end{tabular}

Computer programs: APEX2 and SAINT (Bruker, 2015), SHELXT (Sheldrick, 2015a), SHELXL (Sheldrick, 2015b) and OLEX2 (Dolomanov et al., 2009).

product. The supernatant was removed and the precipitate recrystallized from $40 \mathrm{ml}$ of tert-butyl methyl ether. X-ray quality crystals were obtained in a yield of $1.88 \mathrm{~g}(40 \%)$. Notes: (a) Schlenk techniques are necessary because ynamines and catecholborane are moisture-sensitive; $(b)$ the reaction also works well in diethyl ether, 1,4-dioxane or THF.

${ }^{1} \mathrm{H} \mathrm{NMR}\left(600 \mathrm{MHz}, \mathrm{CDCl}_{3}\right): \delta(\mathrm{ppm})=1.34(d, 6.8 \mathrm{~Hz}, 6 \mathrm{H}$, $\mathrm{CH}_{3}$ of $i$-Pr), 3.89 (hept, $6.8 \mathrm{~Hz}, 1 \mathrm{H}, \mathrm{CH}$ of $i$-Pr), 6.77-6.80 (m, 2H, H5 \& H9), 6.81-6.85 ( $m, 2 \mathrm{H}, \mathrm{H} 7$ \& H15), 6.85-6.92 ( $m$, $6 \mathrm{H}, \mathrm{H} 6$ \& H8 \& H13 \& H14 \& H16 \& H17), 6.95-6.98 ( $m, 2 \mathrm{H}$, $2 \times$ catechol-H), 7.11-7.14 ( $m, 2 \mathrm{H}, 2 \times$ catechol-H), $7.53(s$, 1H, H10).

${ }^{13} \mathrm{C}^{1} \mathrm{H}$ NMR $\left(151 \mathrm{MHz}, \mathrm{CDCl}_{3}\right): \delta(\mathrm{ppm})=22.2(s, \mathrm{C} 1 \&$ C2), 57.4 ( $s, \mathrm{C} 3), 98.1$ ( br s, C11), 111.7 ( $s, 2 \times$ catechol-C), $121.8(s, 2 \times$ catechol-C), $124.3(s, \mathrm{C} 15), 124.6(s, \mathrm{C} 7), 126.3(s$, C5 \& C9), 126.9 ( $s, \mathrm{C} 14$ \& C16), 128.0 ( $s, \mathrm{C} 6$ \& C8), $129.4(s$, $\mathrm{C} 13$ \& C17), 138.8 ( $s, \mathrm{C} 12), 143.3$ ( $s, \mathrm{C} 4), 144.9$ ( $s, \mathrm{C} 10), 148.9$ $(s, \mathrm{C} 18 \& \mathrm{C} 23)$. Inverse gated $\{13\} \mathrm{C}\{1\} \mathrm{H}\} \mathrm{NMR}$ with $D 1=60 \mathrm{~s}$ measured to get integrable ${ }^{13} \mathrm{C}$ NMR.

${ }^{11} \mathrm{~B}$ NMR $\left(160 \mathrm{MHz}, \mathrm{CDCl}_{3}\right): \delta(\mathrm{ppm})=33.2(\mathrm{~s})$.

\section{Refinement}

Crystal data, data collection and structure refinement details are summarized in Table 2. $\mathrm{H}$ atoms were positioned geometrically and refined as riding: $\mathrm{C}-\mathrm{H}=0.95-0.98 \AA$ and $U_{\text {iso }}(\mathrm{H})$ $=1.2 U_{\text {eq }}(\mathrm{C})$ or $1.5 U_{\text {eq }}(\mathrm{C}$-methyl $)$. The absolute structure was not determined because of unreliable Flack and Hooft parameters. 


\section{Acknowledgements}

X-ray services were provided by SMoCC - The Small Molecule Crystallography Center of ETH Zurich. The authors acknowledge Kevin Breitwieser and Nils Trapp for proofreading the manuscript.

\section{Funding information}

Funding for this research was provided by: Schweizerischer Nationalfonds zur Förderung der Wissenschaftlichen Forschung.

\section{References}

Adamczyk-Woźniak, A., Jakubczyk, M., Sporzyński, A. \& Żukowska, G. (2011). Inorg. Chem. Commun. 14, 1753-1755.

Allen, F. H., Watson, D. G., Brammer, L., Orpen, A. G. \& Taylor, R. (2006). International Tables for Crystallography, Vol C, pp. 796811. Chester: IUCr.

Brown, H. C. \& Murray, L. T. (1984). Inorg. Chem. 23, 27462753.

Brown, H. C., Zaidlewicz, M., Dalvi, P. V., Narasimhan, S. \& Mukhopadhyay, A. (1999). Organometallics, 18, 1305-1309.
Buker (2015). APEX2, SAINT and SADABS. Bruker AXS Inc., Madison, Wisconsin, USA.

Dembitsky, V. M., Tolstikov, G. A. \& Srebnik, M. (2002). Eurasian Chem.-Technol. J. 4, 153-167.

Dolomanov, O. V., Bourhis, L. J., Gildea, R. J., Howard, J. A. K. \& Puschmann, H. (2009). J. Appl. Cryst. 42, 339-341.

Goralski, C. T. \& Singaram, B. (2012). ARKIVOC, 2012, 88-113.

Groom, C. R., Bruno, I. J., Lightfoot, M. P. \& Ward, S. C. (2016). Acta Cryst. B72, 171-179.

Hatayama, Y. \& Okuno, T. (2012). Acta Cryst. E68, o84.

Li, M., Li, Y., Zhao, B., Liang, F. \& Jin, L.-Y. (2014). RSC Adv. 4, 30046-30049.

Liu, Y.-L., Kehr, G., Daniliuc, C. G. \& Erker, G. (2017). Chem. Eur. J. 23, 12141-12144.

Sheldrick, G. M. (2015a). Acta Cryst. A71, 3-8.

Sheldrick, G. M. (2015b). Acta Cryst. C71, 3-8.

Viehe, H. G., Janousek, Z., Merényi, R. \& Stella, L. (1985). Acc. Chem. Res. 18, 148-154.

Wang, L., Jian, Z., Daniliuc, C. G., Kehr, G. \& Erker, G. (2018). Dalton Trans. 47, 10853-10856.

Witulski, B., Buschmann, N. \& Bergsträsser, U. (2000). Tetrahedron, 56, 8473-8480.

Zhuo, J.-C., Soloway, A. H., Barnum, B. A. \& Tjarks, W. (2001). Frontiers in Neutron Capture Therapy, edited by M. F. Hawthorne, K. Shelly \& R. J. Wiersema, pp. 785-790. New York: Kluwer Academic/Plenum Publishers. 


\section{supporting information}

Acta Cryst. (2020). E76, 710-714 [https://doi.org/10.1107/S2056989020005289]

\section{Structure of a push-pull olefin prepared by ynamine hydroboration with a borandiol ester}

\section{Joël Gubler and Peter Chen}

Computing details

Data collection: APEX2 (Bruker, 2015); cell refinement: SAINT (Bruker, 2015); data reduction: SAINT (Bruker, 2015); program(s) used to solve structure: SHELXT (Sheldrick, 2015a); program(s) used to refine structure: SHELXL (Sheldrick, 2015b); molecular graphics: OLEX2 (Dolomanov et al., 2009); software used to prepare material for publication: OLEX2 (Dolomanov et al., 2009).

$N-[(Z)-2-(2 H-1,3,2-B e n z o d i o x a b o r o l-2-y l)-2-p h e n y l e t h e n y l]-N$-(propan-2-yl)aniline

\section{Crystal data}

$\mathrm{C}_{23} \mathrm{H}_{22} \mathrm{BNO}_{2}$ $M_{r}=355.22$

Orthorhombic, $P c a 2_{1}$

$a=17.8540(11) \AA$

$b=11.5361(6) \AA$

$c=18.5366(12) \AA$

$V=3817.9(4) \AA^{3}$

$Z=8$

$F(000)=1504$

\section{Data collection}

Bruker-Nonius Kappa APEXII diffractometer

Radiation source: sealed tube Graphite monochromator

Detector resolution: 8.33 pixels $\mathrm{mm}^{-1}$

$\omega$ and $\varphi$ scans

Absorption correction: multi-scan

(SADABS; Bruker, 2015)

$T_{\text {min }}=0.703, T_{\max }=0.733$

Refinement

Refinement on $F^{2}$

Least-squares matrix: full

$R\left[F^{2}>2 \sigma\left(F^{2}\right)\right]=0.039$

$w R\left(F^{2}\right)=0.086$

$S=1.03$

8781 reflections

491 parameters

1 restraint

Primary atom site location: structure-invariant direct methods
$D_{\mathrm{x}}=1.236 \mathrm{Mg} \mathrm{m}^{-3}$

Mo $K \alpha$ radiation, $\lambda=0.71073 \AA$

Cell parameters from 9981 reflections

$\theta=2.5-26.8^{\circ}$

$\mu=0.08 \mathrm{~mm}^{-1}$

$T=100 \mathrm{~K}$

Plate, clear light yellow

$0.38 \times 0.2 \times 0.07 \mathrm{~mm}$

60829 measured reflections

8781 independent reflections

7379 reflections with $I>2 \sigma(I)$

$R_{\text {int }}=0.047$

$\theta_{\max }=27.6^{\circ}, \theta_{\min }=2.8^{\circ}$

$h=-23 \rightarrow 23$

$k=-14 \rightarrow 14$

$l=-24 \rightarrow 24$

Hydrogen site location: inferred from neighbouring sites

$\mathrm{H}$-atom parameters constrained

$w=1 /\left[\sigma^{2}\left(F_{\mathrm{o}}^{2}\right)+(0.0401 P)^{2}+0.7053 P\right]$

where $P=\left(F_{\mathrm{o}}{ }^{2}+2 F_{\mathrm{c}}{ }^{2}\right) / 3$

$(\Delta / \sigma)_{\max }<0.001$

$\Delta \rho_{\max }=0.25$ e $\AA^{-3}$

$\Delta \rho_{\min }=-0.24$ e $\AA^{-3}$ 
Absolute structure: Flack $x$ determined using 3185 quotients $[(\mathrm{I}+)-(\mathrm{I}-)] /[(\mathrm{I}+)+(\mathrm{I}-)]$ (Parsons, Flack and Wagner, Acta Cryst. B69 (2013) 249-259).

Absolute structure parameter: -0.7 (4)

Special details

Geometry. All esds (except the esd in the dihedral angle between two 1.s. planes) are estimated using the full covariance matrix. The cell esds are taken into account individually in the estimation of esds in distances, angles and torsion angles; correlations between esds in cell parameters are only used when they are defined by crystal symmetry. An approximate (isotropic) treatment of cell esds is used for estimating esds involving l.s. planes.

Refinement. 8 reflections were omitted (some are equivalents). These were checked visually and are all results of high background around the beamstop (beginning ice formation or crystalline powder covering the sample). 010 is clearly shadowed by the beamstop. Absolute structure is not claimed due to unreliable Flack and Hooft parameters.

Fractional atomic coordinates and isotropic or equivalent isotropic displacement parameters $\left(\AA^{2}\right)$

\begin{tabular}{|c|c|c|c|c|}
\hline & $x$ & $y$ & $z$ & $U_{\text {iso }} * / U_{\text {eq }}$ \\
\hline O1B & $0.18188(9)$ & $0.59842(13)$ & $0.60028(9)$ & $0.0155(4)$ \\
\hline $\mathrm{O} 2 \mathrm{~B}$ & $0.30086(9)$ & $0.67252(13)$ & $0.61169(9)$ & $0.0158(4)$ \\
\hline N1B & 0.23745 (11) & $0.25154(16)$ & $0.65127(11)$ & 0.0159 (4) \\
\hline $\mathrm{C} 1 \mathrm{~B}$ & $0.10503(15)$ & $0.2002(2)$ & $0.62440(14)$ & $0.0210(6)$ \\
\hline H1BA & 0.0874 & 0.2806 & 0.6251 & $0.032 *$ \\
\hline H1BB & 0.0642 & 0.1485 & 0.6390 & $0.032 *$ \\
\hline H1BC & 0.1215 & 0.1800 & 0.5756 & $0.032 *$ \\
\hline $\mathrm{C} 2 \mathrm{~B}$ & $0.14877(15)$ & $0.2209(2)$ & $0.75303(14)$ & $0.0205(6)$ \\
\hline $\mathrm{H} 2 \mathrm{BA}$ & 0.1928 & 0.2152 & 0.7844 & $0.031 *$ \\
\hline $\mathrm{H} 2 \mathrm{BB}$ & 0.1096 & 0.1686 & 0.7707 & $0.031 *$ \\
\hline $\mathrm{H} 2 \mathrm{BC}$ & 0.1301 & 0.3008 & 0.7533 & $0.031 *$ \\
\hline $\mathrm{C} 3 \mathrm{~B}$ & $0.17032(14)$ & $0.1869(2)$ & $0.67659(13)$ & $0.0160(5)$ \\
\hline H3B & 0.1840 & 0.1028 & 0.6776 & $0.019^{*}$ \\
\hline C4B & $0.30570(13)$ & 0.18933 (19) & $0.64199(14)$ & $0.0147(5)$ \\
\hline C5B & $0.32986(14)$ & $0.1121(2)$ & $0.69452(14)$ & $0.0189(5)$ \\
\hline H5B & 0.3009 & 0.1007 & 0.7369 & $0.023^{*}$ \\
\hline C6B & $0.39606(14)$ & $0.0518(2)$ & $0.68521(16)$ & $0.0230(6)$ \\
\hline H6B & 0.4126 & -0.0006 & 0.7214 & $0.028 *$ \\
\hline C7B & $0.43822(15)$ & $0.0673(2)$ & $0.62365(16)$ & $0.0275(6)$ \\
\hline H7B & 0.4841 & 0.0267 & 0.6177 & $0.033 *$ \\
\hline C8B & $0.41341(15)$ & $0.1423(2)$ & $0.57066(15)$ & $0.0238(6)$ \\
\hline H8B & 0.4420 & 0.1522 & 0.5279 & $0.029 *$ \\
\hline C9B & $0.34704(14)$ & $0.2033(2)$ & $0.57938(14)$ & $0.0191(5)$ \\
\hline H9B & 0.3301 & 0.2543 & 0.5426 & $0.023 *$ \\
\hline $\mathrm{C} 10 \mathrm{~B}$ & $0.23179(13)$ & 0.36795 (19) & $0.63924(13)$ & $0.0151(5)$ \\
\hline H10B & 0.1820 & 0.3949 & 0.6321 & $0.018^{*}$ \\
\hline C11B & $0.28538(13)$ & $0.4526(2)$ & $0.63563(13)$ & $0.0156(5)$ \\
\hline C12B & $0.36555(13)$ & $0.4370(2)$ & $0.65557(13)$ & $0.0142(5)$ \\
\hline C13B & $0.38562(14)$ & $0.3878(2)$ & $0.72178(13)$ & $0.0172(5)$ \\
\hline H13B & 0.3476 & 0.3630 & 0.7541 & $0.021 *$ \\
\hline C14B & $0.46041(15)$ & $0.3748(2)$ & $0.74087(14)$ & $0.0209(5)$ \\
\hline
\end{tabular}




\begin{tabular}{|c|c|c|c|c|}
\hline H14B & 0.4731 & 0.3410 & 0.7860 & $0.025^{*}$ \\
\hline C15B & $0.51658(14)$ & $0.4110(2)$ & $0.69433(14)$ & $0.0210(6)$ \\
\hline H15B & 0.5677 & 0.4012 & 0.7071 & $0.025 *$ \\
\hline $\mathrm{C} 16 \mathrm{~B}$ & 0.49740 (14) & $0.4614(2)$ & $0.62906(14)$ & $0.0188(5)$ \\
\hline H16B & 0.5356 & 0.4869 & 0.5972 & $0.023 *$ \\
\hline C17B & $0.42288(14)$ & 0.4749 (2) & $0.60995(14)$ & $0.0169(5)$ \\
\hline H17B & 0.4106 & 0.5103 & 0.5652 & $0.020 *$ \\
\hline C18B & $0.18063(14)$ & $0.71629(19)$ & $0.58605(13)$ & $0.0140(5)$ \\
\hline C19B & $0.12056(14)$ & $0.7829(2)$ & $0.56544(13)$ & $0.0178(5)$ \\
\hline H19B & 0.0715 & 0.7518 & 0.5614 & $0.021 *$ \\
\hline $\mathrm{C} 20 \mathrm{~B}$ & $0.13606(15)$ & $0.8997(2)$ & $0.55079(14)$ & $0.0198(5)$ \\
\hline $\mathrm{H} 20 \mathrm{~B}$ & 0.0966 & 0.9492 & 0.5356 & $0.024 *$ \\
\hline $\mathrm{C} 21 \mathrm{~B}$ & $0.20801(15)$ & $0.9445(2)$ & $0.55794(13)$ & $0.0192(5)$ \\
\hline $\mathrm{H} 21 \mathrm{~B}$ & 0.2165 & 1.0242 & 0.5478 & $0.023 *$ \\
\hline $\mathrm{C} 22 \mathrm{~B}$ & $0.26793(14)$ & $0.8754(2)$ & $0.57958(13)$ & $0.0179(5)$ \\
\hline $\mathrm{H} 22 \mathrm{~B}$ & 0.3171 & 0.9059 & 0.5849 & $0.022 *$ \\
\hline $\mathrm{C} 23 \mathrm{~B}$ & $0.25211(13)$ & $0.76087(19)$ & $0.59287(13)$ & $0.0151(5)$ \\
\hline B1B & $0.25681(15)$ & $0.5724(2)$ & $0.61526(14)$ & $0.0149(6)$ \\
\hline O1A & $0.59813(9)$ & $-0.09865(13)$ & $0.40292(10)$ & $0.0176(4)$ \\
\hline $\mathrm{O} 2 \mathrm{~A}$ & $0.71747(9)$ & $-0.17253(13)$ & $0.39845(9)$ & $0.0174(4)$ \\
\hline N1A & $0.65302(11)$ & $0.24387(17)$ & $0.34366(11)$ & $0.0161(4)$ \\
\hline $\mathrm{C} 1 \mathrm{~A}$ & $0.52189(14)$ & $0.2942(2)$ & $0.37802(15)$ & $0.0228(6)$ \\
\hline H1AA & 0.5416 & 0.3104 & 0.4263 & $0.034 *$ \\
\hline $\mathrm{H} 1 \mathrm{AB}$ & 0.5027 & 0.2146 & 0.3764 & $0.034 *$ \\
\hline $\mathrm{H} 1 \mathrm{AC}$ & 0.4813 & 0.3485 & 0.3671 & $0.034^{*}$ \\
\hline $\mathrm{C} 2 \mathrm{~A}$ & $0.58421(14)$ & $0.3081(2)$ & $0.32256(14)$ & $0.0177(5)$ \\
\hline $\mathrm{H} 2 \mathrm{~A}$ & 0.5976 & 0.3923 & 0.3213 & $0.021 *$ \\
\hline $\mathrm{C} 3 \mathrm{~A}$ & 0.55869 (14) & $0.2754(2)$ & $0.24731(15)$ & $0.0227(6)$ \\
\hline H3AA & 0.5407 & 0.1952 & 0.2473 & $0.034 *$ \\
\hline $\mathrm{H} 3 \mathrm{AB}$ & 0.6008 & 0.2829 & 0.2137 & $0.034 *$ \\
\hline $\mathrm{H} 3 \mathrm{AC}$ & 0.5180 & 0.3272 & 0.2323 & $0.034^{*}$ \\
\hline $\mathrm{C} 4 \mathrm{~A}$ & $0.72025(13)$ & $0.3083(2)$ & $0.35237(13)$ & $0.0139(5)$ \\
\hline $\mathrm{C} 5 \mathrm{~A}$ & $0.76423(13)$ & $0.2910(2)$ & $0.41332(13)$ & $0.0150(5)$ \\
\hline H5A & 0.7496 & 0.2358 & 0.4487 & $0.018^{*}$ \\
\hline C6A & $0.82952(14)$ & $0.3543(2)$ & 0.42245 (14) & $0.0199(5)$ \\
\hline H6A & 0.8596 & 0.3423 & 0.4640 & $0.024^{*}$ \\
\hline C7A & $0.85112(15)$ & $0.4353(2)$ & $0.37098(15)$ & $0.0233(6)$ \\
\hline H7A & 0.8964 & 0.4776 & 0.3768 & $0.028 *$ \\
\hline C8A & $0.80630(14)$ & $0.4541(2)$ & $0.31124(16)$ & $0.0227(6)$ \\
\hline H8A & 0.8204 & 0.5105 & 0.2765 & $0.027^{*}$ \\
\hline C9A & $0.74113(14)$ & $0.3912(2)$ & 0.30178 (14) & $0.0182(5)$ \\
\hline H9A & 0.7106 & 0.4047 & 0.2607 & $0.022 *$ \\
\hline $\mathrm{C} 10 \mathrm{~A}$ & 0.64818 (14) & $0.1276(2)$ & $0.35658(13)$ & $0.0157(5)$ \\
\hline $\mathrm{H} 10 \mathrm{~A}$ & 0.5984 & 0.1001 & 0.3624 & $0.019^{*}$ \\
\hline $\mathrm{C} 11 \mathrm{~A}$ & $0.70180(13)$ & 0.0433 (2) & $0.36266(12)$ & $0.0136(5)$ \\
\hline $\mathrm{C} 12 \mathrm{~A}$ & $0.78275(13)$ & $0.0582(2)$ & $0.34518(13)$ & $0.0143(5)$ \\
\hline $\mathrm{C} 13 \mathrm{~A}$ & 0.83799 (13) & $0.0207(2)$ & $0.39292(13)$ & $0.0159(5)$ \\
\hline H13A & 0.8239 & -0.0154 & 0.4369 & $0.019 *$ \\
\hline
\end{tabular}




$\begin{array}{lllll}\text { C14A } & 0.91359(14) & 0.0353(2) & 0.37700(15) & 0.0184(5) \\ \text { H14A } & 0.9505 & 0.0104 & 0.4105 & 0.022^{*} \\ \text { C15A } & 0.93517(15) & 0.0860(2) & 0.31277(14) & 0.0209(6) \\ \text { H15A } & 0.9868 & 0.0967 & 0.3021 & 0.025^{*} \\ \text { C16A } & 0.88076(14) & 0.1212(2) & 0.26390(14) & 0.0209(6) \\ \text { H16A } & 0.8952 & 0.1549 & 0.2193 & 0.025^{*} \\ \text { C17A } & 0.80555(14) & 0.1074(2) & 0.27994(13) & 0.0166(5) \\ \text { H17A } & 0.7689 & 0.1317 & 0.2460 & 0.020^{*} \\ \text { C18A } & 0.59638(14) & -0.2147(2) & 0.42114(13) & 0.0155(5) \\ \text { C19A } & 0.53579(14) & -0.2820(2) & 0.43916(14) & 0.0196(5) \\ \text { H19A } & 0.4864 & -0.2514 & 0.4402 & 0.024^{*} \\ \text { C20A } & 0.55064(15) & -0.3974(2) & 0.45574(14) & 0.0212(6) \\ \text { H20A } & 0.5102 & -0.4469 & 0.4685 & 0.025^{*} \\ \text { C21A } & 0.62240(15) & -0.4420(2) & 0.45425(14) & 0.0221(6) \\ \text { H21A } & 0.6302 & -0.5212 & 0.4661 & 0.027^{*} \\ \text { C22A } & 0.68384(15) & -0.3727(2) & 0.43561(14) & 0.0215(6) \\ \text { H22A } & 0.7335 & -0.4024 & 0.4346 & 0.026^{*} \\ \text { C23A } & 0.66814(14) & -0.2591(2) & 0.41882(13) & 0.0159(5) \\ \text { B1A } & 0.67343(15) & -0.0740(2) & 0.38806(15) & 0.0149(5)\end{array}$

Atomic displacement parameters $\left(\AA^{2}\right)$

\begin{tabular}{lllllll}
\hline & $U^{11}$ & $U^{22}$ & $U^{33}$ & $U^{12}$ & $U^{13}$ & $U^{23}$ \\
\hline O1B & $0.0139(8)$ & $0.0119(8)$ & $0.0206(9)$ & $0.0005(7)$ & $0.0001(7)$ & $0.0023(7)$ \\
O2B & $0.0137(8)$ & $0.0144(8)$ & $0.0194(9)$ & $-0.0010(6)$ & $-0.0018(7)$ & $0.0016(7)$ \\
N1B & $0.0115(10)$ & $0.0142(9)$ & $0.0218(11)$ & $0.0004(8)$ & $0.0030(8)$ & $0.0028(8)$ \\
C1B & $0.0183(13)$ & $0.0203(12)$ & $0.0245(15)$ & $-0.0017(10)$ & $-0.0016(11)$ & $-0.0009(11)$ \\
C2B & $0.0194(14)$ & $0.0215(13)$ & $0.0206(13)$ & $-0.0021(11)$ & $0.0031(11)$ & $0.0012(10)$ \\
C3B & $0.0142(13)$ & $0.0135(11)$ & $0.0205(13)$ & $-0.0017(10)$ & $0.0030(10)$ & $0.0013(10)$ \\
C4B & $0.0115(12)$ & $0.0112(10)$ & $0.0213(13)$ & $-0.0010(9)$ & $-0.0012(10)$ & $-0.0023(10)$ \\
C5B & $0.0187(13)$ & $0.0162(12)$ & $0.0218(14)$ & $-0.0021(10)$ & $-0.0029(10)$ & $0.0006(10)$ \\
C6B & $0.0174(13)$ & $0.0157(12)$ & $0.0360(16)$ & $0.0007(10)$ & $-0.0082(12)$ & $0.0023(11)$ \\
C7B & $0.0144(13)$ & $0.0196(12)$ & $0.0487(19)$ & $0.0027(11)$ & $0.0015(13)$ & $-0.0046(12)$ \\
C8B & $0.0196(14)$ & $0.0191(13)$ & $0.0328(16)$ & $-0.0020(11)$ & $0.0108(11)$ & $-0.0065(11)$ \\
C9B & $0.0173(13)$ & $0.0168(12)$ & $0.0232(13)$ & $-0.0015(10)$ & $0.0015(10)$ & $-0.0014(10)$ \\
C10B & $0.0132(12)$ & $0.0174(11)$ & $0.0147(11)$ & $0.0022(9)$ & $0.0007(9)$ & $0.0013(10)$ \\
C11B & $0.0146(12)$ & $0.0169(11)$ & $0.0152(12)$ & $0.0014(10)$ & $0.0027(10)$ & $0.0006(10)$ \\
C12B & $0.0133(12)$ & $0.0120(11)$ & $0.0173(12)$ & $0.0018(9)$ & $0.0002(10)$ & $-0.0012(10)$ \\
C13B & $0.0182(13)$ & $0.0161(12)$ & $0.0172(13)$ & $0.0000(10)$ & $0.0021(10)$ & $-0.0014(10)$ \\
C14B & $0.0217(14)$ & $0.0217(13)$ & $0.0195(13)$ & $0.0023(11)$ & $-0.0053(11)$ & $0.0007(11)$ \\
C15B & $0.0143(13)$ & $0.0208(12)$ & $0.0281(15)$ & $0.0023(10)$ & $-0.0057(11)$ & $-0.0022(11)$ \\
C16B & $0.0144(12)$ & $0.0178(12)$ & $0.0244(14)$ & $-0.0007(10)$ & $0.0032(10)$ & $-0.0005(10)$ \\
C17B & $0.0177(12)$ & $0.0163(11)$ & $0.0169(12)$ & $0.0007(10)$ & $0.0008(10)$ & $0.0008(10)$ \\
C18B & $0.0171(12)$ & $0.0124(11)$ & $0.0124(11)$ & $-0.0024(10)$ & $0.0031(9)$ & $0.0000(9)$ \\
C19B & $0.0140(13)$ & $0.0202(13)$ & $0.0191(13)$ & $0.0031(10)$ & $0.0011(10)$ & $0.0001(10)$ \\
C20B & $0.0236(14)$ & $0.0186(12)$ & $0.0171(13)$ & $0.0061(11)$ & $0.0014(11)$ & $0.0014(10)$ \\
C21B & $0.0277(14)$ & $0.0125(11)$ & $0.0172(13)$ & $0.0003(11)$ & $0.0044(11)$ & $-0.0002(10)$ \\
C22B & $0.0217(13)$ & $0.0174(11)$ & $0.0147(12)$ & $-0.0032(10)$ & $0.0000(10)$ & $-0.0025(9)$
\end{tabular}




\begin{tabular}{|c|c|c|c|c|c|c|}
\hline C23B & $0.0158(12)$ & $0.0167(11)$ & $0.0127(11)$ & $0.0028(10)$ & -0.0025 (9) & $-0.0023(10)$ \\
\hline B1B & $0.0128(13)$ & $0.0191(13)$ & $0.0129(13)$ & $-0.0004(11)$ & $0.0016(11)$ & $-0.0006(11)$ \\
\hline O1A & $0.0134(9)$ & $0.0138(8)$ & $0.0258(10)$ & $-0.0012(7)$ & $0.0003(7)$ & $0.0027(7)$ \\
\hline $\mathrm{O} 2 \mathrm{~A}$ & $0.0139(9)$ & $0.0146(8)$ & $0.0237(10)$ & $-0.0005(7)$ & $0.0028(7)$ & $0.0023(7)$ \\
\hline N1A & $0.0116(10)$ & $0.0147(10)$ & $0.0220(11)$ & $0.0002(8)$ & $-0.0036(8)$ & $0.0030(8)$ \\
\hline $\mathrm{C} 1 \mathrm{~A}$ & $0.0161(13)$ & $0.0221(13)$ & $0.0304(15)$ & $0.0042(11)$ & $0.0001(11)$ & 0.0005 (12) \\
\hline $\mathrm{C} 2 \mathrm{~A}$ & $0.0128(12)$ & $0.0150(11)$ & $0.0251(14)$ & $0.0015(10)$ & $-0.0032(10)$ & $0.0033(10)$ \\
\hline $\mathrm{C} 3 \mathrm{~A}$ & $0.0154(14)$ & $0.0273(14)$ & $0.0254(14)$ & $0.0027(11)$ & $-0.0061(11)$ & $0.0047(11)$ \\
\hline $\mathrm{C} 4 \mathrm{~A}$ & $0.0121(12)$ & $0.0119(11)$ & $0.0175(12)$ & $0.0009(9)$ & $0.0005(10)$ & $-0.0018(9)$ \\
\hline $\mathrm{C} 5 \mathrm{~A}$ & $0.0157(12)$ & $0.0142(10)$ & $0.0151(12)$ & $0.0010(9)$ & $0.0010(10)$ & $-0.0004(9)$ \\
\hline C6A & $0.0160(12)$ & $0.0214(12)$ & $0.0223(14)$ & $0.0021(10)$ & $-0.0038(10)$ & $-0.0035(11)$ \\
\hline C7A & 0.0155 (13) & $0.0179(12)$ & $0.0366(16)$ & $-0.0054(11)$ & $0.0001(12)$ & $-0.0006(11)$ \\
\hline C8A & 0.0198 (14) & $0.0182(12)$ & $0.0300(15)$ & $-0.0014(11)$ & $0.0034(11)$ & $0.0066(11)$ \\
\hline C9A & 0.0175 (13) & $0.0173(12)$ & $0.0198(13)$ & $0.0018(10)$ & $-0.0009(10)$ & $0.0033(10)$ \\
\hline $\mathrm{C} 10 \mathrm{~A}$ & $0.0129(12)$ & $0.0171(12)$ & $0.0172(12)$ & $-0.0036(10)$ & $-0.0012(9)$ & $0.0010(10)$ \\
\hline C11A & $0.0125(12)$ & $0.0146(11)$ & $0.0138(12)$ & $0.0000(9)$ & $-0.0014(9)$ & $0.0005(9)$ \\
\hline $\mathrm{C} 12 \mathrm{~A}$ & $0.0143(12)$ & $0.0123(11)$ & $0.0164(12)$ & $-0.0009(9)$ & $0.0007(10)$ & $-0.0042(10)$ \\
\hline C13A & $0.0162(12)$ & $0.0143(11)$ & $0.0170(13)$ & $0.0002(10)$ & $0.0011(10)$ & $0.0002(10)$ \\
\hline C14A & $0.0140(12)$ & $0.0166(12)$ & $0.0247(14)$ & $0.0000(10)$ & $-0.0032(10)$ & $-0.0033(10)$ \\
\hline $\mathrm{C} 15 \mathrm{~A}$ & $0.0152(13)$ & $0.0220(12)$ & $0.0255(14)$ & $-0.0017(10)$ & $0.0056(11)$ & $-0.0028(11)$ \\
\hline $\mathrm{C} 16 \mathrm{~A}$ & 0.0229 (14) & $0.0215(13)$ & $0.0182(13)$ & $-0.0034(11)$ & $0.0064(11)$ & $0.0000(11)$ \\
\hline $\mathrm{C} 17 \mathrm{~A}$ & 0.0165 (12) & $0.0167(12)$ & $0.0166(12)$ & $-0.0003(10)$ & $-0.0014(10)$ & $-0.0008(10)$ \\
\hline C18A & $0.0185(13)$ & $0.0154(11)$ & $0.0127(12)$ & $-0.0018(10)$ & $-0.0012(9)$ & $0.0004(9)$ \\
\hline C19A & $0.0151(13)$ & $0.0228(13)$ & $0.0211(13)$ & $-0.0022(11)$ & $-0.0002(10)$ & $0.0028(11)$ \\
\hline $\mathrm{C} 20 \mathrm{~A}$ & $0.0236(14)$ & $0.0209(13)$ & $0.0190(13)$ & $-0.0103(11)$ & $-0.0021(11)$ & $0.0036(10)$ \\
\hline $\mathrm{C} 21 \mathrm{~A}$ & $0.0294(15)$ & $0.0155(12)$ & $0.0215(13)$ & $-0.0025(11)$ & $-0.0005(11)$ & $0.0047(10)$ \\
\hline $\mathrm{C} 22 \mathrm{~A}$ & $0.0216(14)$ & $0.0191(12)$ & $0.0237(14)$ & $0.0030(11)$ & $-0.0002(11)$ & $0.0019(11)$ \\
\hline $\mathrm{C} 23 \mathrm{~A}$ & $0.0137(12)$ & $0.0178(12)$ & $0.0164(12)$ & $-0.0037(10)$ & $0.0009(10)$ & $0.0009(10)$ \\
\hline B1A & $0.0126(13)$ & $0.0177(13)$ & $0.0143(13)$ & $-0.0006(11)$ & $0.0002(11)$ & $-0.0026(11)$ \\
\hline
\end{tabular}

Geometric parameters $(\AA, \stackrel{\circ}{)})$

\begin{tabular}{|c|c|c|c|}
\hline $\mathrm{O} 1 \mathrm{~B}-\mathrm{C} 18 \mathrm{~B}$ & $1.385(3)$ & $\mathrm{O} 1 \mathrm{~A}-\mathrm{C} 18 \mathrm{~A}$ & $1.382(3)$ \\
\hline $\mathrm{O} 1 \mathrm{~B}-\mathrm{B} 1 \mathrm{~B}$ & $1.399(3)$ & $\mathrm{O} 1 \mathrm{~A}-\mathrm{B} 1 \mathrm{~A}$ & $1.402(3)$ \\
\hline $\mathrm{O} 2 \mathrm{~B}-\mathrm{C} 23 \mathrm{~B}$ & $1.385(3)$ & $\mathrm{O} 2 \mathrm{~A}-\mathrm{C} 23 \mathrm{~A}$ & $1.384(3)$ \\
\hline $\mathrm{O} 2 \mathrm{~B}-\mathrm{B} 1 \mathrm{~B}$ & $1.398(3)$ & $\mathrm{O} 2 \mathrm{~A}-\mathrm{B} 1 \mathrm{~A}$ & $1.396(3)$ \\
\hline $\mathrm{N} 1 \mathrm{~B}-\mathrm{C} 3 \mathrm{~B}$ & $1.488(3)$ & $\mathrm{N} 1 \mathrm{~A}-\mathrm{C} 2 \mathrm{~A}$ & $1.487(3)$ \\
\hline $\mathrm{N} 1 \mathrm{~B}-\mathrm{C} 4 \mathrm{~B}$ & $1.425(3)$ & $\mathrm{N} 1 \mathrm{~A}-\mathrm{C} 4 \mathrm{~A}$ & $1.421(3)$ \\
\hline $\mathrm{N} 1 \mathrm{~B}-\mathrm{C} 10 \mathrm{~B}$ & $1.365(3)$ & $\mathrm{N} 1 \mathrm{~A}-\mathrm{C} 10 \mathrm{~A}$ & $1.365(3)$ \\
\hline $\mathrm{C} 1 \mathrm{~B}-\mathrm{H} 1 \mathrm{BA}$ & 0.9800 & $\mathrm{C} 1 \mathrm{~A}-\mathrm{H} 1 \mathrm{AA}$ & 0.9800 \\
\hline $\mathrm{C} 1 \mathrm{~B}-\mathrm{H} 1 \mathrm{BB}$ & 0.9800 & $\mathrm{C} 1 \mathrm{~A}-\mathrm{H} 1 \mathrm{AB}$ & 0.9800 \\
\hline $\mathrm{C} 1 \mathrm{~B}-\mathrm{H} 1 \mathrm{BC}$ & 0.9800 & $\mathrm{C} 1 \mathrm{~A}-\mathrm{H} 1 \mathrm{AC}$ & 0.9800 \\
\hline $\mathrm{C} 1 \mathrm{~B}-\mathrm{C} 3 \mathrm{~B}$ & $1.523(4)$ & $\mathrm{C} 1 \mathrm{~A}-\mathrm{C} 2 \mathrm{~A}$ & $1.523(4)$ \\
\hline $\mathrm{C} 2 \mathrm{~B}-\mathrm{H} 2 \mathrm{BA}$ & 0.9800 & $\mathrm{C} 2 \mathrm{~A}-\mathrm{H} 2 \mathrm{~A}$ & 1.0000 \\
\hline $\mathrm{C} 2 \mathrm{~B}-\mathrm{H} 2 \mathrm{BB}$ & 0.9800 & $\mathrm{C} 2 \mathrm{~A}-\mathrm{C} 3 \mathrm{~A}$ & $1.515(4)$ \\
\hline $\mathrm{C} 2 \mathrm{~B}-\mathrm{H} 2 \mathrm{BC}$ & 0.9800 & $\mathrm{C} 3 \mathrm{~A}-\mathrm{H} 3 \mathrm{AA}$ & 0.9800 \\
\hline $\mathrm{C} 2 \mathrm{~B}-\mathrm{C} 3 \mathrm{~B}$ & $1.520(3)$ & $\mathrm{C} 3 \mathrm{~A}-\mathrm{H} 3 \mathrm{AB}$ & 0.9800 \\
\hline $\mathrm{C} 3 \mathrm{~B}-\mathrm{H} 3 \mathrm{~B}$ & 1.0000 & $\mathrm{C} 3 \mathrm{~A}-\mathrm{H} 3 \mathrm{AC}$ & 0.9800 \\
\hline
\end{tabular}




\begin{tabular}{|c|c|c|c|}
\hline $\mathrm{C} 4 \mathrm{~B}-\mathrm{C} 5 \mathrm{~B}$ & $1.389(3)$ & $\mathrm{C} 4 \mathrm{~A}-\mathrm{C} 5 \mathrm{~A}$ & $1.390(3)$ \\
\hline $\mathrm{C} 4 \mathrm{~B}-\mathrm{C} 9 \mathrm{~B}$ & $1.385(4)$ & $\mathrm{C} 4 \mathrm{~A}-\mathrm{C} 9 \mathrm{~A}$ & $1.390(3)$ \\
\hline $\mathrm{C} 5 \mathrm{~B}-\mathrm{H} 5 \mathrm{~B}$ & 0.9500 & $\mathrm{C} 5 \mathrm{~A}-\mathrm{H} 5 \mathrm{~A}$ & 0.9500 \\
\hline $\mathrm{C} 5 \mathrm{~B}-\mathrm{C} 6 \mathrm{~B}$ & $1.382(3)$ & $\mathrm{C} 5 \mathrm{~A}-\mathrm{C} 6 \mathrm{~A}$ & $1.386(3)$ \\
\hline $\mathrm{C} 6 \mathrm{~B}-\mathrm{H} 6 \mathrm{~B}$ & 0.9500 & C6A-H6A & 0.9500 \\
\hline $\mathrm{C} 6 \mathrm{~B}-\mathrm{C} 7 \mathrm{~B}$ & $1.379(4)$ & $\mathrm{C} 6 \mathrm{~A}-\mathrm{C} 7 \mathrm{~A}$ & $1.390(4)$ \\
\hline C7B-H7B & 0.9500 & $\mathrm{C} 7 \mathrm{~A}-\mathrm{H} 7 \mathrm{~A}$ & 0.9500 \\
\hline $\mathrm{C} 7 \mathrm{~B}-\mathrm{C} 8 \mathrm{~B}$ & $1.382(4)$ & $\mathrm{C} 7 \mathrm{~A}-\mathrm{C} 8 \mathrm{~A}$ & $1.383(4)$ \\
\hline $\mathrm{C} 8 \mathrm{~B}-\mathrm{H} 8 \mathrm{~B}$ & 0.9500 & $\mathrm{C} 8 \mathrm{~A}-\mathrm{H} 8 \mathrm{~A}$ & 0.9500 \\
\hline $\mathrm{C} 8 \mathrm{~B}-\mathrm{C} 9 \mathrm{~B}$ & $1.387(4)$ & $\mathrm{C} 8 \mathrm{~A}-\mathrm{C} 9 \mathrm{~A}$ & $1.382(4)$ \\
\hline C9B-H9B & 0.9500 & C9A-H9A & 0.9500 \\
\hline $\mathrm{C} 10 \mathrm{~B}-\mathrm{H} 10 \mathrm{~B}$ & 0.9500 & $\mathrm{C} 10 \mathrm{~A}-\mathrm{H} 10 \mathrm{~A}$ & 0.9500 \\
\hline $\mathrm{C} 10 \mathrm{~B}-\mathrm{C} 11 \mathrm{~B}$ & $1.369(3)$ & $\mathrm{C} 10 \mathrm{~A}-\mathrm{C} 11 \mathrm{~A}$ & $1.370(3)$ \\
\hline $\mathrm{C} 11 \mathrm{~B}-\mathrm{C} 12 \mathrm{~B}$ & $1.489(3)$ & $\mathrm{C} 11 \mathrm{~A}-\mathrm{C} 12 \mathrm{~A}$ & $1.491(3)$ \\
\hline $\mathrm{C} 11 \mathrm{~B}-\mathrm{B} 1 \mathrm{~B}$ & $1.521(4)$ & $\mathrm{C} 11 \mathrm{~A}-\mathrm{B} 1 \mathrm{~A}$ & $1.519(3)$ \\
\hline $\mathrm{C} 12 \mathrm{~B}-\mathrm{C} 13 \mathrm{~B}$ & $1.399(3)$ & $\mathrm{C} 12 \mathrm{~A}-\mathrm{C} 13 \mathrm{~A}$ & $1.394(3)$ \\
\hline $\mathrm{C} 12 \mathrm{~B}-\mathrm{C} 17 \mathrm{~B}$ & $1.398(3)$ & $\mathrm{C} 12 \mathrm{~A}-\mathrm{C} 17 \mathrm{~A}$ & $1.397(3)$ \\
\hline $\mathrm{C} 13 \mathrm{~B}-\mathrm{H} 13 \mathrm{~B}$ & 0.9500 & $\mathrm{C} 13 \mathrm{~A}-\mathrm{H} 13 \mathrm{~A}$ & 0.9500 \\
\hline $\mathrm{C} 13 \mathrm{~B}-\mathrm{C} 14 \mathrm{~B}$ & $1.389(4)$ & $\mathrm{C} 13 \mathrm{~A}-\mathrm{C} 14 \mathrm{~A}$ & $1.392(3)$ \\
\hline $\mathrm{C} 14 \mathrm{~B}-\mathrm{H} 14 \mathrm{~B}$ & 0.9500 & $\mathrm{C} 14 \mathrm{~A}-\mathrm{H} 14 \mathrm{~A}$ & 0.9500 \\
\hline $\mathrm{C} 14 \mathrm{~B}-\mathrm{C} 15 \mathrm{~B}$ & $1.387(4)$ & $\mathrm{C} 14 \mathrm{~A}-\mathrm{C} 15 \mathrm{~A}$ & $1.381(4)$ \\
\hline $\mathrm{C} 15 \mathrm{~B}-\mathrm{H} 15 \mathrm{~B}$ & 0.9500 & $\mathrm{C} 15 \mathrm{~A}-\mathrm{H} 15 \mathrm{~A}$ & 0.9500 \\
\hline $\mathrm{C} 15 \mathrm{~B}-\mathrm{C} 16 \mathrm{~B}$ & $1.385(4)$ & $\mathrm{C} 15 \mathrm{~A}-\mathrm{C} 16 \mathrm{~A}$ & $1.389(4)$ \\
\hline $\mathrm{C} 16 \mathrm{~B}-\mathrm{H} 16 \mathrm{~B}$ & 0.9500 & $\mathrm{C} 16 \mathrm{~A}-\mathrm{H} 16 \mathrm{~A}$ & 0.9500 \\
\hline $\mathrm{C} 16 \mathrm{~B}-\mathrm{C} 17 \mathrm{~B}$ & $1.386(3)$ & $\mathrm{C} 16 \mathrm{~A}-\mathrm{C} 17 \mathrm{~A}$ & $1.385(3)$ \\
\hline $\mathrm{C} 17 \mathrm{~B}-\mathrm{H} 17 \mathrm{~B}$ & 0.9500 & $\mathrm{C} 17 \mathrm{~A}-\mathrm{H} 17 \mathrm{~A}$ & 0.9500 \\
\hline $\mathrm{C} 18 \mathrm{~B}-\mathrm{C} 19 \mathrm{~B}$ & $1.374(3)$ & $\mathrm{C} 18 \mathrm{~A}-\mathrm{C} 19 \mathrm{~A}$ & $1.372(3)$ \\
\hline $\mathrm{C} 18 \mathrm{~B}-\mathrm{C} 23 \mathrm{~B}$ & $1.382(3)$ & $\mathrm{C} 18 \mathrm{~A}-\mathrm{C} 23 \mathrm{~A}$ & $1.381(3)$ \\
\hline $\mathrm{C} 19 \mathrm{~B}-\mathrm{H} 19 \mathrm{~B}$ & 0.9500 & C19A-H19A & 0.9500 \\
\hline $\mathrm{C} 19 \mathrm{~B}-\mathrm{C} 20 \mathrm{~B}$ & $1.401(3)$ & $\mathrm{C} 20 \mathrm{~A}-\mathrm{H} 20 \mathrm{~A}$ & 0.9500 \\
\hline $\mathrm{C} 20 \mathrm{~B}-\mathrm{H} 20 \mathrm{~B}$ & 0.9500 & $\mathrm{C} 20 \mathrm{~A}-\mathrm{C} 21 \mathrm{~A}$ & $1.381(4)$ \\
\hline $\mathrm{C} 20 \mathrm{~B}-\mathrm{C} 21 \mathrm{~B}$ & $1.391(4)$ & $\mathrm{C} 21 \mathrm{~A}-\mathrm{H} 21 \mathrm{~A}$ & 0.9500 \\
\hline $\mathrm{C} 21 \mathrm{~B}-\mathrm{H} 21 \mathrm{~B}$ & 0.9500 & $\mathrm{C} 21 \mathrm{~A}-\mathrm{C} 22 \mathrm{~A}$ & $1.401(4)$ \\
\hline $\mathrm{C} 21 \mathrm{~B}-\mathrm{C} 22 \mathrm{~B}$ & $1.393(4)$ & $\mathrm{C} 22 \mathrm{~A}-\mathrm{H} 22 \mathrm{~A}$ & 0.9500 \\
\hline $\mathrm{C} 22 \mathrm{~B}-\mathrm{H} 22 \mathrm{~B}$ & 0.9500 & $\mathrm{C} 22 \mathrm{~A}-\mathrm{C} 23 \mathrm{~A}$ & $1.375(3)$ \\
\hline $\mathrm{C} 22 \mathrm{~B}-\mathrm{C} 23 \mathrm{~B}$ & $1.374(3)$ & & \\
\hline $\mathrm{C} 18 \mathrm{~B}-\mathrm{O} 1 \mathrm{~B}-\mathrm{B} 1 \mathrm{~B}$ & $105.28(18)$ & $\mathrm{C} 18 \mathrm{~A}-\mathrm{O} 1 \mathrm{~A}-\mathrm{B} 1 \mathrm{~A}$ & $105.46(18)$ \\
\hline $\mathrm{C} 23 \mathrm{~B}-\mathrm{O} 2 \mathrm{~B}-\mathrm{B} 1 \mathrm{~B}$ & $105.43(18)$ & $\mathrm{C} 23 \mathrm{~A}-\mathrm{O} 2 \mathrm{~A}-\mathrm{B} 1 \mathrm{~A}$ & $105.50(18)$ \\
\hline $\mathrm{C} 4 \mathrm{~B}-\mathrm{N} 1 \mathrm{~B}-\mathrm{C} 3 \mathrm{~B}$ & $118.34(18)$ & $\mathrm{C} 4 \mathrm{~A}-\mathrm{N} 1 \mathrm{~A}-\mathrm{C} 2 \mathrm{~A}$ & $117.83(18)$ \\
\hline $\mathrm{C} 10 \mathrm{~B}-\mathrm{N} 1 \mathrm{~B}-\mathrm{C} 3 \mathrm{~B}$ & $119.02(19)$ & $\mathrm{C} 10 \mathrm{~A}-\mathrm{N} 1 \mathrm{~A}-\mathrm{C} 2 \mathrm{~A}$ & $118.9(2)$ \\
\hline $\mathrm{C} 10 \mathrm{~B}-\mathrm{N} 1 \mathrm{~B}-\mathrm{C} 4 \mathrm{~B}$ & $122.6(2)$ & $\mathrm{C} 10 \mathrm{~A}-\mathrm{N} 1 \mathrm{~A}-\mathrm{C} 4 \mathrm{~A}$ & $123.2(2)$ \\
\hline $\mathrm{H} 1 \mathrm{BA}-\mathrm{C} 1 \mathrm{~B}-\mathrm{H} 1 \mathrm{BB}$ & 109.5 & $\mathrm{H} 1 \mathrm{AA}-\mathrm{C} 1 \mathrm{~A}-\mathrm{H} 1 \mathrm{AB}$ & 109.5 \\
\hline $\mathrm{H} 1 \mathrm{BA}-\mathrm{C} 1 \mathrm{~B}-\mathrm{H} 1 \mathrm{BC}$ & 109.5 & $\mathrm{H} 1 \mathrm{AA}-\mathrm{C} 1 \mathrm{~A}-\mathrm{H} 1 \mathrm{AC}$ & 109.5 \\
\hline $\mathrm{H} 1 \mathrm{BB}-\mathrm{C} 1 \mathrm{~B}-\mathrm{H} 1 \mathrm{BC}$ & 109.5 & $\mathrm{H} 1 \mathrm{AB}-\mathrm{C} 1 \mathrm{~A}-\mathrm{H} 1 \mathrm{AC}$ & 109.5 \\
\hline $\mathrm{C} 3 \mathrm{~B}-\mathrm{C} 1 \mathrm{~B}-\mathrm{H} 1 \mathrm{BA}$ & 109.5 & $\mathrm{C} 2 \mathrm{~A}-\mathrm{C} 1 \mathrm{~A}-\mathrm{H} 1 \mathrm{AA}$ & 109.5 \\
\hline $\mathrm{C} 3 \mathrm{~B}-\mathrm{C} 1 \mathrm{~B}-\mathrm{H} 1 \mathrm{BB}$ & 109.5 & $\mathrm{C} 2 \mathrm{~A}-\mathrm{C} 1 \mathrm{~A}-\mathrm{H} 1 \mathrm{AB}$ & 109.5 \\
\hline $\mathrm{C} 3 \mathrm{~B}-\mathrm{C} 1 \mathrm{~B}-\mathrm{H} 1 \mathrm{BC}$ & 109.5 & $\mathrm{C} 2 \mathrm{~A}-\mathrm{C} 1 \mathrm{~A}-\mathrm{H} 1 \mathrm{AC}$ & 109.5 \\
\hline
\end{tabular}




$\begin{array}{ll}\text { H2BA-C2B-H2BB } & 109.5 \\ \text { H2BA-C2B-H2BC } & 109.5 \\ \text { H2BB-C2B-H2BC } & 109.5 \\ \text { C3B-C2B-H2BA } & 109.5 \\ \text { C3B-C2B-H2BB } & 109.5 \\ \text { C3B-C2B-H2BC } & 109.5 \\ \text { N1B-C3B-C1B } & 111.5(2) \\ \text { N1B-C3B-C2B } & 111.6(2) \\ \text { N1B-C3B-H3B } & 107.2 \\ \text { C1B-C3B-H3B } & 107.2 \\ \text { C2B-C3B-C1B } & 111.9(2) \\ \text { C2B-C3B-H3B } & 107.2 \\ \text { C5B-C4B-N1B } & 120.3(2) \\ \text { C9B-C4B-N1B } & 119.9(2) \\ \text { C9B-C4B-C5B } & 119.8(2) \\ \text { C4B-C5B-H5B } & 120.0 \\ \text { C6B-C5B-C4B } & 120.1(2) \\ \text { C6B-C5B-H5B } & 120.0 \\ \text { C5B-C6B-H6B } & 119.8 \\ \text { C7B-C6B-C5B } & 120.3(2) \\ \text { C7B-C6B-H6B } & 119.8 \\ \text { C6B-C7B-H7B } & 120.2 \\ \text { C6B-C7B-C8B } & 119.7(2) \\ \text { C8B-C7B-H7B } & 120.2 \\ \text { C7B-C8B-H8B } & 119.7 \\ \text { C7B-C8B-C9B } & 120.5(2) \\ \text { C9B-C8B-H8B } & 119.7 \\ \text { C4B-C9B-C8B } & 119.6(2) \\ \text { C4B-C9B-H9B } & 120.2 \\ \text { C8B-C9B-H9B } & 120.2 \\ \text { N1B-C10B-H10B } & 114.4 \\ \text { N1B-C10B-C11B } & 131.2(2) \\ \text { C11B-C10B-H10B } & 114.4 \\ \text { C10B-C11B-C12B } & 125.0(2) \\ \text { C10B-C11B-B1B } & 115.2(2) \\ \text { C12B-C11B-B1B } & 119.6(2) \\ \text { C13B-C12B-C11B } & 120.9(2) \\ \text { C17B-C12B-C11B } & 121.1(2) \\ \text { C17B-C12B-C13B } & 118.0(2) \\ \text { C12B-C13B-H13B } & 119.6 \\ \text { C14B-C13B-C12B } & 120.9(2) \\ \text { C14B-C13B-H13B } & 119.6 \\ \text { C13B-C14B-H14B } & 119.9 \\ \text { C15B-C14B-C13B } & 120.3(2) \\ \text { C15B-C14B-H14B } & 119.9 \\ \text { C14B-C15B-H15B } & \\ \text { C16B-C15B-C14B } & \\ \text { C16B-C15B-H15B } & \\ & \end{array}$

$\begin{array}{ll}\mathrm{N} 1 \mathrm{~A}-\mathrm{C} 2 \mathrm{~A}-\mathrm{C} 1 \mathrm{~A} & 111.9(2) \\ \mathrm{N} 1 \mathrm{~A}-\mathrm{C} 2 \mathrm{~A}-\mathrm{H} 2 \mathrm{~A} & 107.0 \\ \mathrm{~N} 1 \mathrm{~A}-\mathrm{C} 2 \mathrm{~A}-\mathrm{C} 3 \mathrm{~A} & 111.5(2) \\ \mathrm{C} 1 \mathrm{~A}-\mathrm{C} 2 \mathrm{~A}-\mathrm{H} 2 \mathrm{~A} & 107.0 \\ \mathrm{C} 3 \mathrm{~A}-\mathrm{C} 2 \mathrm{~A}-\mathrm{C} 1 \mathrm{~A} & 112.1(2) \\ \mathrm{C} 3 \mathrm{~A}-\mathrm{C} 2 \mathrm{~A}-\mathrm{H} 2 \mathrm{~A} & 107.0 \\ \mathrm{C} 2 \mathrm{~A}-\mathrm{C} 3 \mathrm{~A}-\mathrm{H} 3 \mathrm{AA} & 109.5 \\ \mathrm{C} 2 \mathrm{~A}-\mathrm{C} 3 \mathrm{~A}-\mathrm{H} 3 \mathrm{AB} & 109.5 \\ \mathrm{C} 2 \mathrm{~A}-\mathrm{C} 3 \mathrm{~A}-\mathrm{H} 3 \mathrm{AC} & 109.5 \\ \mathrm{H} 3 \mathrm{AA}-\mathrm{C} 3 \mathrm{~A}-\mathrm{H} 3 \mathrm{AB} & 109.5 \\ \mathrm{H} 3 \mathrm{AA}-\mathrm{C} 3 \mathrm{~A}-\mathrm{H} 3 \mathrm{AC} & 109.5 \\ \mathrm{H} 3 \mathrm{AB}-\mathrm{C} 3 \mathrm{~A}-\mathrm{H} 3 \mathrm{AC} & 109.5 \\ \mathrm{C} 5 \mathrm{~A}-\mathrm{C} 4 \mathrm{~A}-\mathrm{N} 1 \mathrm{~A} & 119.6(2) \\ \mathrm{C} 5 \mathrm{~A}-\mathrm{C} 4 \mathrm{~A}-\mathrm{C} 9 \mathrm{~A} & 119.7(2) \\ \mathrm{C} 9 \mathrm{~A}-\mathrm{C} 4 \mathrm{~A}-\mathrm{N} 1 \mathrm{~A} & 120.6(2) \\ \mathrm{C} 4 \mathrm{~A}-\mathrm{C} 5 \mathrm{~A}-\mathrm{HA} & 120.1\end{array}$

$\mathrm{C} 4 \mathrm{~A}-\mathrm{C} 5 \mathrm{~A}-\mathrm{H} 5 \mathrm{~A}$

$\mathrm{C} 6 \mathrm{~A}-\mathrm{C} 5 \mathrm{~A}-\mathrm{C} 4 \mathrm{~A}$

$\mathrm{C} 6 \mathrm{~A}-\mathrm{C} 5 \mathrm{~A}-\mathrm{H} 5 \mathrm{~A}$

120.1

119.9 (2)

120.1

119.9

$120.3(2)$

119.9

120.2

$119.6(2)$

120.2

119.8

$120.4(2)$

119.8

120.0

120.1 (2)

120.0

114.0

$131.9(2)$

114.0

$125.3(2)$

$115.1(2)$

$119.5(2)$

$120.8(2)$

$118.0(2)$

$121.2(2)$

119.5

120.9 (2)

119.5

119.8

$120.3(2)$

119.8

120.3

119.4 (2)

120.3 


\begin{tabular}{|c|c|c|c|}
\hline $\mathrm{C} 15 \mathrm{~B}-\mathrm{C} 16 \mathrm{~B}-\mathrm{H} 16 \mathrm{~B}$ & 119.7 & $\mathrm{C} 15 \mathrm{~A}-\mathrm{C} 16 \mathrm{~A}-\mathrm{H} 16 \mathrm{~A}$ & 119.9 \\
\hline $\mathrm{C} 15 \mathrm{~B}-\mathrm{C} 16 \mathrm{~B}-\mathrm{C} 17 \mathrm{~B}$ & $120.5(2)$ & $\mathrm{C} 17 \mathrm{~A}-\mathrm{C} 16 \mathrm{~A}-\mathrm{C} 15 \mathrm{~A}$ & $120.3(2)$ \\
\hline $\mathrm{C} 17 \mathrm{~B}-\mathrm{C} 16 \mathrm{~B}-\mathrm{H} 16 \mathrm{~B}$ & 119.7 & $\mathrm{C} 17 \mathrm{~A}-\mathrm{C} 16 \mathrm{~A}-\mathrm{H} 16 \mathrm{~A}$ & 119.9 \\
\hline $\mathrm{C} 12 \mathrm{~B}-\mathrm{C} 17 \mathrm{~B}-\mathrm{H} 17 \mathrm{~B}$ & 119.5 & $\mathrm{C} 12 \mathrm{~A}-\mathrm{C} 17 \mathrm{~A}-\mathrm{H} 17 \mathrm{~A}$ & 119.5 \\
\hline $\mathrm{C} 16 \mathrm{~B}-\mathrm{C} 17 \mathrm{~B}-\mathrm{C} 12 \mathrm{~B}$ & $120.9(2)$ & $\mathrm{C} 16 \mathrm{~A}-\mathrm{C} 17 \mathrm{~A}-\mathrm{C} 12 \mathrm{~A}$ & $121.0(2)$ \\
\hline $\mathrm{C} 16 \mathrm{~B}-\mathrm{C} 17 \mathrm{~B}-\mathrm{H} 17 \mathrm{~B}$ & 119.5 & $\mathrm{C} 16 \mathrm{~A}-\mathrm{C} 17 \mathrm{~A}-\mathrm{H} 17 \mathrm{~A}$ & 119.5 \\
\hline $\mathrm{C} 19 \mathrm{~B}-\mathrm{C} 18 \mathrm{~B}-\mathrm{O} 1 \mathrm{~B}$ & $127.9(2)$ & $\mathrm{C} 19 \mathrm{~A}-\mathrm{C} 18 \mathrm{~A}-\mathrm{O} 1 \mathrm{~A}$ & $128.7(2)$ \\
\hline $\mathrm{C} 19 \mathrm{~B}-\mathrm{C} 18 \mathrm{~B}-\mathrm{C} 23 \mathrm{~B}$ & $122.6(2)$ & $\mathrm{C} 19 \mathrm{~A}-\mathrm{C} 18 \mathrm{~A}-\mathrm{C} 23 \mathrm{~A}$ & $122.0(2)$ \\
\hline $\mathrm{C} 23 \mathrm{~B}-\mathrm{C} 18 \mathrm{~B}-\mathrm{O} 1 \mathrm{~B}$ & $109.4(2)$ & $\mathrm{C} 23 \mathrm{~A}-\mathrm{C} 18 \mathrm{~A}-\mathrm{O} 1 \mathrm{~A}$ & $109.3(2)$ \\
\hline $\mathrm{C} 18 \mathrm{~B}-\mathrm{C} 19 \mathrm{~B}-\mathrm{H} 19 \mathrm{~B}$ & 122.0 & $\mathrm{C} 18 \mathrm{~A}-\mathrm{C} 19 \mathrm{~A}-\mathrm{H} 19 \mathrm{~A}$ & 121.8 \\
\hline $\mathrm{C} 18 \mathrm{~B}-\mathrm{C} 19 \mathrm{~B}-\mathrm{C} 20 \mathrm{~B}$ & $115.9(2)$ & $\mathrm{C} 18 \mathrm{~A}-\mathrm{C} 19 \mathrm{~A}-\mathrm{C} 20 \mathrm{~A}$ & $116.4(2)$ \\
\hline $\mathrm{C} 20 \mathrm{~B}-\mathrm{C} 19 \mathrm{~B}-\mathrm{H} 19 \mathrm{~B}$ & 122.0 & $\mathrm{C} 20 \mathrm{~A}-\mathrm{C} 19 \mathrm{~A}-\mathrm{H} 19 \mathrm{~A}$ & 121.8 \\
\hline $\mathrm{C} 19 \mathrm{~B}-\mathrm{C} 20 \mathrm{~B}-\mathrm{H} 20 \mathrm{~B}$ & 119.3 & $\mathrm{C} 19 \mathrm{~A}-\mathrm{C} 20 \mathrm{~A}-\mathrm{H} 20 \mathrm{~A}$ & 119.0 \\
\hline $\mathrm{C} 21 \mathrm{~B}-\mathrm{C} 20 \mathrm{~B}-\mathrm{C} 19 \mathrm{~B}$ & $121.4(2)$ & $\mathrm{C} 21 \mathrm{~A}-\mathrm{C} 20 \mathrm{~A}-\mathrm{C} 19 \mathrm{~A}$ & $121.9(2)$ \\
\hline $\mathrm{C} 21 \mathrm{~B}-\mathrm{C} 20 \mathrm{~B}-\mathrm{H} 20 \mathrm{~B}$ & 119.3 & $\mathrm{C} 21 \mathrm{~A}-\mathrm{C} 20 \mathrm{~A}-\mathrm{H} 20 \mathrm{~A}$ & 119.0 \\
\hline $\mathrm{C} 20 \mathrm{~B}-\mathrm{C} 21 \mathrm{~B}-\mathrm{H} 21 \mathrm{~B}$ & 119.2 & $\mathrm{C} 20 \mathrm{~A}-\mathrm{C} 21 \mathrm{~A}-\mathrm{H} 21 \mathrm{~A}$ & 119.4 \\
\hline $\mathrm{C} 20 \mathrm{~B}-\mathrm{C} 21 \mathrm{~B}-\mathrm{C} 22 \mathrm{~B}$ & $121.6(2)$ & $\mathrm{C} 20 \mathrm{~A}-\mathrm{C} 21 \mathrm{~A}-\mathrm{C} 22 \mathrm{~A}$ & $121.3(2)$ \\
\hline $\mathrm{C} 22 \mathrm{~B}-\mathrm{C} 21 \mathrm{~B}-\mathrm{H} 21 \mathrm{~B}$ & 119.2 & $\mathrm{C} 22 \mathrm{~A}-\mathrm{C} 21 \mathrm{~A}-\mathrm{H} 21 \mathrm{~A}$ & 119.4 \\
\hline $\mathrm{C} 21 \mathrm{~B}-\mathrm{C} 22 \mathrm{~B}-\mathrm{H} 22 \mathrm{~B}$ & 121.8 & $\mathrm{C} 21 \mathrm{~A}-\mathrm{C} 22 \mathrm{~A}-\mathrm{H} 22 \mathrm{~A}$ & 122.0 \\
\hline $\mathrm{C} 23 \mathrm{~B}-\mathrm{C} 22 \mathrm{~B}-\mathrm{C} 21 \mathrm{~B}$ & $116.4(2)$ & $\mathrm{C} 23 \mathrm{~A}-\mathrm{C} 22 \mathrm{~A}-\mathrm{C} 21 \mathrm{~A}$ & $116.1(2)$ \\
\hline $\mathrm{C} 23 \mathrm{~B}-\mathrm{C} 22 \mathrm{~B}-\mathrm{H} 22 \mathrm{~B}$ & 121.8 & $\mathrm{C} 23 \mathrm{~A}-\mathrm{C} 22 \mathrm{~A}-\mathrm{H} 22 \mathrm{~A}$ & 122.0 \\
\hline $\mathrm{C} 18 \mathrm{~B}-\mathrm{C} 23 \mathrm{~B}-\mathrm{O} 2 \mathrm{~B}$ & $109.24(19)$ & $\mathrm{C} 18 \mathrm{~A}-\mathrm{C} 23 \mathrm{~A}-\mathrm{O} 2 \mathrm{~A}$ & $109.3(2)$ \\
\hline $\mathrm{C} 22 \mathrm{~B}-\mathrm{C} 23 \mathrm{~B}-\mathrm{O} 2 \mathrm{~B}$ & $128.6(2)$ & $\mathrm{C} 22 \mathrm{~A}-\mathrm{C} 23 \mathrm{~A}-\mathrm{O} 2 \mathrm{~A}$ & $128.3(2)$ \\
\hline $\mathrm{C} 22 \mathrm{~B}-\mathrm{C} 23 \mathrm{~B}-\mathrm{C} 18 \mathrm{~B}$ & $122.1(2)$ & $\mathrm{C} 22 \mathrm{~A}-\mathrm{C} 23 \mathrm{~A}-\mathrm{C} 18 \mathrm{~A}$ & $122.4(2)$ \\
\hline $\mathrm{O} 1 \mathrm{~B}-\mathrm{B} 1 \mathrm{~B}-\mathrm{C} 11 \mathrm{~B}$ & $124.4(2)$ & $\mathrm{O} 1 \mathrm{~A}-\mathrm{B} 1 \mathrm{~A}-\mathrm{C} 11 \mathrm{~A}$ & $124.2(2)$ \\
\hline $\mathrm{O} 2 \mathrm{~B}-\mathrm{B} 1 \mathrm{~B}-\mathrm{O} 1 \mathrm{~B}$ & $110.6(2)$ & $\mathrm{O} 2 \mathrm{~A}-\mathrm{B} 1 \mathrm{~A}-\mathrm{O} 1 \mathrm{~A}$ & $110.4(2)$ \\
\hline $\mathrm{O} 2 \mathrm{~B}-\mathrm{B} 1 \mathrm{~B}-\mathrm{C} 11 \mathrm{~B}$ & $125.0(2)$ & $\mathrm{O} 2 \mathrm{~A}-\mathrm{B} 1 \mathrm{~A}-\mathrm{C} 11 \mathrm{~A}$ & $125.5(2)$ \\
\hline $\mathrm{O} 1 \mathrm{~B}-\mathrm{C} 18 \mathrm{~B}-\mathrm{C} 19 \mathrm{~B}-\mathrm{C} 20 \mathrm{~B}$ & $-176.7(2)$ & $\mathrm{O} 1 \mathrm{~A}-\mathrm{C} 18 \mathrm{~A}-\mathrm{C} 19 \mathrm{~A}-\mathrm{C} 20 \mathrm{~A}$ & $-179.5(2)$ \\
\hline $\mathrm{O} 1 \mathrm{~B}-\mathrm{C} 18 \mathrm{~B}-\mathrm{C} 23 \mathrm{~B}-\mathrm{O} 2 \mathrm{~B}$ & $0.0(3)$ & $\mathrm{O} 1 \mathrm{~A}-\mathrm{C} 18 \mathrm{~A}-\mathrm{C} 23 \mathrm{~A}-\mathrm{O} 2 \mathrm{~A}$ & $-0.5(3)$ \\
\hline $\mathrm{O} 1 \mathrm{~B}-\mathrm{C} 18 \mathrm{~B}-\mathrm{C} 23 \mathrm{~B}-\mathrm{C} 22 \mathrm{~B}$ & $177.9(2)$ & $\mathrm{O} 1 \mathrm{~A}-\mathrm{C} 18 \mathrm{~A}-\mathrm{C} 23 \mathrm{~A}-\mathrm{C} 22 \mathrm{~A}$ & $178.9(2)$ \\
\hline $\mathrm{N} 1 \mathrm{~B}-\mathrm{C} 4 \mathrm{~B}-\mathrm{C} 5 \mathrm{~B}-\mathrm{C} 6 \mathrm{~B}$ & $179.9(2)$ & $\mathrm{N} 1 \mathrm{~A}-\mathrm{C} 4 \mathrm{~A}-\mathrm{C} 5 \mathrm{~A}-\mathrm{C} 6 \mathrm{~A}$ & $-179.4(2)$ \\
\hline $\mathrm{N} 1 \mathrm{~B}-\mathrm{C} 4 \mathrm{~B}-\mathrm{C} 9 \mathrm{~B}-\mathrm{C} 8 \mathrm{~B}$ & $-179.9(2)$ & $\mathrm{N} 1 \mathrm{~A}-\mathrm{C} 4 \mathrm{~A}-\mathrm{C} 9 \mathrm{~A}-\mathrm{C} 8 \mathrm{~A}$ & $179.4(2)$ \\
\hline $\mathrm{N} 1 \mathrm{~B}-\mathrm{C} 10 \mathrm{~B}-\mathrm{C} 11 \mathrm{~B}-\mathrm{C} 12 \mathrm{~B}$ & $-9.7(4)$ & $\mathrm{N} 1 \mathrm{~A}-\mathrm{C} 10 \mathrm{~A}-\mathrm{C} 11 \mathrm{~A}-\mathrm{C} 12 \mathrm{~A}$ & $-9.7(4)$ \\
\hline $\mathrm{N} 1 \mathrm{~B}-\mathrm{C} 10 \mathrm{~B}-\mathrm{C} 11 \mathrm{~B}-\mathrm{B} 1 \mathrm{~B}$ & $175.5(2)$ & $\mathrm{N} 1 \mathrm{~A}-\mathrm{C} 10 \mathrm{~A}-\mathrm{C} 11 \mathrm{~A}-\mathrm{B} 1 \mathrm{~A}$ & $171.6(2)$ \\
\hline $\mathrm{C} 3 \mathrm{~B}-\mathrm{N} 1 \mathrm{~B}-\mathrm{C} 4 \mathrm{~B}-\mathrm{C} 5 \mathrm{~B}$ & $-45.6(3)$ & $\mathrm{C} 2 \mathrm{~A}-\mathrm{N} 1 \mathrm{~A}-\mathrm{C} 4 \mathrm{~A}-\mathrm{C} 5 \mathrm{~A}$ & $132.7(2)$ \\
\hline $\mathrm{C} 3 \mathrm{~B}-\mathrm{N} 1 \mathrm{~B}-\mathrm{C} 4 \mathrm{~B}-\mathrm{C} 9 \mathrm{~B}$ & $132.5(2)$ & $\mathrm{C} 2 \mathrm{~A}-\mathrm{N} 1 \mathrm{~A}-\mathrm{C} 4 \mathrm{~A}-\mathrm{C} 9 \mathrm{~A}$ & $-45.1(3)$ \\
\hline $\mathrm{C} 3 \mathrm{~B}-\mathrm{N} 1 \mathrm{~B}-\mathrm{C} 10 \mathrm{~B}-\mathrm{C} 11 \mathrm{~B}$ & $159.6(3)$ & $\mathrm{C} 2 \mathrm{~A}-\mathrm{N} 1 \mathrm{~A}-\mathrm{C} 10 \mathrm{~A}-\mathrm{C} 11 \mathrm{~A}$ & $165.3(2)$ \\
\hline $\mathrm{C} 4 \mathrm{~B}-\mathrm{N} 1 \mathrm{~B}-\mathrm{C} 3 \mathrm{~B}-\mathrm{C} 1 \mathrm{~B}$ & $-123.7(2)$ & $\mathrm{C} 4 \mathrm{~A}-\mathrm{N} 1 \mathrm{~A}-\mathrm{C} 2 \mathrm{~A}-\mathrm{C} 1 \mathrm{~A}$ & $-120.9(2)$ \\
\hline $\mathrm{C} 4 \mathrm{~B}-\mathrm{N} 1 \mathrm{~B}-\mathrm{C} 3 \mathrm{~B}-\mathrm{C} 2 \mathrm{~B}$ & $110.3(2)$ & $\mathrm{C} 4 \mathrm{~A}-\mathrm{N} 1 \mathrm{~A}-\mathrm{C} 2 \mathrm{~A}-\mathrm{C} 3 \mathrm{~A}$ & $112.7(2)$ \\
\hline $\mathrm{C} 4 \mathrm{~B}-\mathrm{N} 1 \mathrm{~B}-\mathrm{C} 10 \mathrm{~B}-\mathrm{C} 11 \mathrm{~B}$ & $-19.1(4)$ & $\mathrm{C} 4 \mathrm{~A}-\mathrm{N} 1 \mathrm{~A}-\mathrm{C} 10 \mathrm{~A}-\mathrm{C} 11 \mathrm{~A}$ & $-17.5(4)$ \\
\hline $\mathrm{C} 4 \mathrm{~B}-\mathrm{C} 5 \mathrm{~B}-\mathrm{C} 6 \mathrm{~B}-\mathrm{C} 7 \mathrm{~B}$ & $-0.4(4)$ & $\mathrm{C} 4 \mathrm{~A}-\mathrm{C} 5 \mathrm{~A}-\mathrm{C} 6 \mathrm{~A}-\mathrm{C} 7 \mathrm{~A}$ & $0.1(4)$ \\
\hline $\mathrm{C} 5 \mathrm{~B}-\mathrm{C} 4 \mathrm{~B}-\mathrm{C} 9 \mathrm{~B}-\mathrm{C} 8 \mathrm{~B}$ & $-1.8(4)$ & $\mathrm{C} 5 \mathrm{~A}-\mathrm{C} 4 \mathrm{~A}-\mathrm{C} 9 \mathrm{~A}-\mathrm{C} 8 \mathrm{~A}$ & $1.6(4)$ \\
\hline $\mathrm{C} 5 \mathrm{~B}-\mathrm{C} 6 \mathrm{~B}-\mathrm{C} 7 \mathrm{~B}-\mathrm{C} 8 \mathrm{~B}$ & $-1.0(4)$ & $\mathrm{C} 5 \mathrm{~A}-\mathrm{C} 6 \mathrm{~A}-\mathrm{C} 7 \mathrm{~A}-\mathrm{C} 8 \mathrm{~A}$ & $1.3(4)$ \\
\hline $\mathrm{C} 6 \mathrm{~B}-\mathrm{C} 7 \mathrm{~B}-\mathrm{C} 8 \mathrm{~B}-\mathrm{C} 9 \mathrm{~B}$ & $1.0(4)$ & $\mathrm{C} 6 \mathrm{~A}-\mathrm{C} 7 \mathrm{~A}-\mathrm{C} 8 \mathrm{~A}-\mathrm{C} 9 \mathrm{~A}$ & $-1.3(4)$ \\
\hline $\mathrm{C} 7 \mathrm{~B}-\mathrm{C} 8 \mathrm{~B}-\mathrm{C} 9 \mathrm{~B}-\mathrm{C} 4 \mathrm{~B}$ & $0.5(4)$ & $\mathrm{C} 7 \mathrm{~A}-\mathrm{C} 8 \mathrm{~A}-\mathrm{C} 9 \mathrm{~A}-\mathrm{C} 4 \mathrm{~A}$ & $-0.1(4)$ \\
\hline $\mathrm{C} 9 \mathrm{~B}-\mathrm{C} 4 \mathrm{~B}-\mathrm{C} 5 \mathrm{~B}-\mathrm{C} 6 \mathrm{~B}$ & $1.8(4)$ & $\mathrm{C} 9 \mathrm{~A}-\mathrm{C} 4 \mathrm{~A}-\mathrm{C} 5 \mathrm{~A}-\mathrm{C} 6 \mathrm{~A}$ & $-1.6(4)$ \\
\hline $\mathrm{C} 10 \mathrm{~B}-\mathrm{N} 1 \mathrm{~B}-\mathrm{C} 3 \mathrm{~B}-\mathrm{C} 1 \mathrm{~B}$ & $57.5(3)$ & $\mathrm{C} 10 \mathrm{~A}-\mathrm{N} 1 \mathrm{~A}-\mathrm{C} 2 \mathrm{~A}-\mathrm{C} 1 \mathrm{~A}$ & $56.4(3)$ \\
\hline
\end{tabular}




\begin{tabular}{|c|c|c|c|}
\hline $\mathrm{C} 10 \mathrm{~B}-\mathrm{N} 1 \mathrm{~B}-\mathrm{C} 3 \mathrm{~B}-\mathrm{C} 2 \mathrm{~B}$ & $-68.4(3)$ & $\mathrm{C} 10 \mathrm{~A}-\mathrm{N} 1 \mathrm{~A}-\mathrm{C} 2 \mathrm{~A}-\mathrm{C} 3 \mathrm{~A}$ & $-69.9(3)$ \\
\hline $\mathrm{C} 10 \mathrm{~B}-\mathrm{N} 1 \mathrm{~B}-\mathrm{C} 4 \mathrm{~B}-\mathrm{C} 5 \mathrm{~B}$ & $133.1(2)$ & $\mathrm{C} 10 \mathrm{~A}-\mathrm{N} 1 \mathrm{~A}-\mathrm{C} 4 \mathrm{~A}-\mathrm{C} 5 \mathrm{~A}$ & $-44.5(3)$ \\
\hline $\mathrm{C} 10 \mathrm{~B}-\mathrm{N} 1 \mathrm{~B}-\mathrm{C} 4 \mathrm{~B}-\mathrm{C} 9 \mathrm{~B}$ & $-48.8(3)$ & $\mathrm{C} 10 \mathrm{~A}-\mathrm{N} 1 \mathrm{~A}-\mathrm{C} 4 \mathrm{~A}-\mathrm{C} 9 \mathrm{~A}$ & $137.6(2)$ \\
\hline $\mathrm{C} 10 \mathrm{~B}-\mathrm{C} 11 \mathrm{~B}-\mathrm{C} 12 \mathrm{~B}-\mathrm{C} 13 \mathrm{~B}$ & $-50.5(3)$ & $\mathrm{C} 10 \mathrm{~A}-\mathrm{C} 11 \mathrm{~A}-\mathrm{C} 12 \mathrm{~A}-\mathrm{C} 13 \mathrm{~A}$ & $132.2(3)$ \\
\hline $\mathrm{C} 10 \mathrm{~B}-\mathrm{C} 11 \mathrm{~B}-\mathrm{C} 12 \mathrm{~B}-\mathrm{C} 17 \mathrm{~B}$ & $131.9(3)$ & $\mathrm{C} 10 \mathrm{~A}-\mathrm{C} 11 \mathrm{~A}-\mathrm{C} 12 \mathrm{~A}-\mathrm{C} 17 \mathrm{~A}$ & $-49.3(3)$ \\
\hline $\mathrm{C} 10 \mathrm{~B}-\mathrm{C} 11 \mathrm{~B}-\mathrm{B} 1 \mathrm{~B}-\mathrm{O} 1 \mathrm{~B}$ & $-0.5(4)$ & $\mathrm{C} 10 \mathrm{~A}-\mathrm{C} 11 \mathrm{~A}-\mathrm{B} 1 \mathrm{~A}-\mathrm{O} 1 \mathrm{~A}$ & $1.7(4)$ \\
\hline $\mathrm{C} 10 \mathrm{~B}-\mathrm{C} 11 \mathrm{~B}-\mathrm{B} 1 \mathrm{~B}-\mathrm{O} 2 \mathrm{~B}$ & $176.9(2)$ & $\mathrm{C} 10 \mathrm{~A}-\mathrm{C} 11 \mathrm{~A}-\mathrm{B} 1 \mathrm{~A}-\mathrm{O} 2 \mathrm{~A}$ & $-179.8(2)$ \\
\hline $\mathrm{C} 11 \mathrm{~B}-\mathrm{C} 12 \mathrm{~B}-\mathrm{C} 13 \mathrm{~B}-\mathrm{C} 14 \mathrm{~B}$ & $-179.0(2)$ & $\mathrm{C} 11 \mathrm{~A}-\mathrm{C} 12 \mathrm{~A}-\mathrm{C} 13 \mathrm{~A}-\mathrm{C} 14 \mathrm{~A}$ & $-179.5(2)$ \\
\hline $\mathrm{C} 11 \mathrm{~B}-\mathrm{C} 12 \mathrm{~B}-\mathrm{C} 17 \mathrm{~B}-\mathrm{C} 16 \mathrm{~B}$ & $179.3(2)$ & $\mathrm{C} 11 \mathrm{~A}-\mathrm{C} 12 \mathrm{~A}-\mathrm{C} 17 \mathrm{~A}-\mathrm{C} 16 \mathrm{~A}$ & $179.9(2)$ \\
\hline $\mathrm{C} 12 \mathrm{~B}-\mathrm{C} 11 \mathrm{~B}-\mathrm{B} 1 \mathrm{~B}-\mathrm{O} 1 \mathrm{~B}$ & $-175.6(2)$ & $\mathrm{C} 12 \mathrm{~A}-\mathrm{C} 11 \mathrm{~A}-\mathrm{B} 1 \mathrm{~A}-\mathrm{O} 1 \mathrm{~A}$ & $-177.2(2)$ \\
\hline $\mathrm{C} 12 \mathrm{~B}-\mathrm{C} 11 \mathrm{~B}-\mathrm{B} 1 \mathrm{~B}-\mathrm{O} 2 \mathrm{~B}$ & $1.7(4)$ & $\mathrm{C} 12 \mathrm{~A}-\mathrm{C} 11 \mathrm{~A}-\mathrm{B} 1 \mathrm{~A}-\mathrm{O} 2 \mathrm{~A}$ & $1.4(4)$ \\
\hline $\mathrm{C} 12 \mathrm{~B}-\mathrm{C} 13 \mathrm{~B}-\mathrm{C} 14 \mathrm{~B}-\mathrm{C} 15 \mathrm{~B}$ & $0.2(4)$ & $\mathrm{C} 12 \mathrm{~A}-\mathrm{C} 13 \mathrm{~A}-\mathrm{C} 14 \mathrm{~A}-\mathrm{C} 15 \mathrm{~A}$ & $-1.0(4)$ \\
\hline $\mathrm{C} 13 \mathrm{~B}-\mathrm{C} 12 \mathrm{~B}-\mathrm{C} 17 \mathrm{~B}-\mathrm{C} 16 \mathrm{~B}$ & $1.6(3)$ & $\mathrm{C} 13 \mathrm{~A}-\mathrm{C} 12 \mathrm{~A}-\mathrm{C} 17 \mathrm{~A}-\mathrm{C} 16 \mathrm{~A}$ & $-1.6(3)$ \\
\hline $\mathrm{C} 13 \mathrm{~B}-\mathrm{C} 14 \mathrm{~B}-\mathrm{C} 15 \mathrm{~B}-\mathrm{C} 16 \mathrm{~B}$ & $0.8(4)$ & $\mathrm{C} 13 \mathrm{~A}-\mathrm{C} 14 \mathrm{~A}-\mathrm{C} 15 \mathrm{~A}-\mathrm{C} 16 \mathrm{~A}$ & $-0.6(4)$ \\
\hline $\mathrm{C} 14 \mathrm{~B}-\mathrm{C} 15 \mathrm{~B}-\mathrm{C} 16 \mathrm{~B}-\mathrm{C} 17 \mathrm{~B}$ & $-0.5(4)$ & $\mathrm{C} 14 \mathrm{~A}-\mathrm{C} 15 \mathrm{~A}-\mathrm{C} 16 \mathrm{~A}-\mathrm{C} 17 \mathrm{~A}$ & $1.1(4)$ \\
\hline $\mathrm{C} 15 \mathrm{~B}-\mathrm{C} 16 \mathrm{~B}-\mathrm{C} 17 \mathrm{~B}-\mathrm{C} 12 \mathrm{~B}$ & $-0.6(4)$ & $\mathrm{C} 15 \mathrm{~A}-\mathrm{C} 16 \mathrm{~A}-\mathrm{C} 17 \mathrm{~A}-\mathrm{C} 12 \mathrm{~A}$ & $0.1(4)$ \\
\hline $\mathrm{C} 17 \mathrm{~B}-\mathrm{C} 12 \mathrm{~B}-\mathrm{C} 13 \mathrm{~B}-\mathrm{C} 14 \mathrm{~B}$ & $-1.3(3)$ & $\mathrm{C} 17 \mathrm{~A}-\mathrm{C} 12 \mathrm{~A}-\mathrm{C} 13 \mathrm{~A}-\mathrm{C} 14 \mathrm{~A}$ & $2.0(3)$ \\
\hline $\mathrm{C} 18 \mathrm{~B}-\mathrm{O} 1 \mathrm{~B}-\mathrm{B} 1 \mathrm{~B}-\mathrm{O} 2 \mathrm{~B}$ & $1.1(3)$ & $\mathrm{C} 18 \mathrm{~A}-\mathrm{O} 1 \mathrm{~A}-\mathrm{B} 1 \mathrm{~A}-\mathrm{O} 2 \mathrm{~A}$ & $-1.2(3)$ \\
\hline $\mathrm{C} 18 \mathrm{~B}-\mathrm{O} 1 \mathrm{~B}-\mathrm{B} 1 \mathrm{~B}-\mathrm{C} 11 \mathrm{~B}$ & $178.7(2)$ & $\mathrm{C} 18 \mathrm{~A}-\mathrm{O} 1 \mathrm{~A}-\mathrm{B} 1 \mathrm{~A}-\mathrm{C} 11 \mathrm{~A}$ & $177.5(2)$ \\
\hline $\mathrm{C} 18 \mathrm{~B}-\mathrm{C} 19 \mathrm{~B}-\mathrm{C} 20 \mathrm{~B}-\mathrm{C} 21 \mathrm{~B}$ & $-0.9(4)$ & $\mathrm{C} 18 \mathrm{~A}-\mathrm{C} 19 \mathrm{~A}-\mathrm{C} 20 \mathrm{~A}-\mathrm{C} 21 \mathrm{~A}$ & $0.1(4)$ \\
\hline $\mathrm{C} 19 \mathrm{~B}-\mathrm{C} 18 \mathrm{~B}-\mathrm{C} 23 \mathrm{~B}-\mathrm{O} 2 \mathrm{~B}$ & $-177.8(2)$ & $\mathrm{C} 19 \mathrm{~A}-\mathrm{C} 18 \mathrm{~A}-\mathrm{C} 23 \mathrm{~A}-\mathrm{O} 2 \mathrm{~A}$ & $179.4(2)$ \\
\hline $\mathrm{C} 19 \mathrm{~B}-\mathrm{C} 18 \mathrm{~B}-\mathrm{C} 23 \mathrm{~B}-\mathrm{C} 22 \mathrm{~B}$ & $0.1(4)$ & $\mathrm{C} 19 \mathrm{~A}-\mathrm{C} 18 \mathrm{~A}-\mathrm{C} 23 \mathrm{~A}-\mathrm{C} 22 \mathrm{~A}$ & $-1.2(4)$ \\
\hline $\mathrm{C} 19 \mathrm{~B}-\mathrm{C} 20 \mathrm{~B}-\mathrm{C} 21 \mathrm{~B}-\mathrm{C} 22 \mathrm{~B}$ & $0.3(4)$ & $\mathrm{C} 19 \mathrm{~A}-\mathrm{C} 20 \mathrm{~A}-\mathrm{C} 21 \mathrm{~A}-\mathrm{C} 22 \mathrm{~A}$ & $-0.2(4)$ \\
\hline $\mathrm{C} 20 \mathrm{~B}-\mathrm{C} 21 \mathrm{~B}-\mathrm{C} 22 \mathrm{~B}-\mathrm{C} 23 \mathrm{~B}$ & $0.5(4)$ & $\mathrm{C} 20 \mathrm{~A}-\mathrm{C} 21 \mathrm{~A}-\mathrm{C} 22 \mathrm{~A}-\mathrm{C} 23 \mathrm{~A}$ & $-0.3(4)$ \\
\hline $\mathrm{C} 21 \mathrm{~B}-\mathrm{C} 22 \mathrm{~B}-\mathrm{C} 23 \mathrm{~B}-\mathrm{O} 2 \mathrm{~B}$ & $176.8(2)$ & $\mathrm{C} 21 \mathrm{~A}-\mathrm{C} 22 \mathrm{~A}-\mathrm{C} 23 \mathrm{~A}-\mathrm{O} 2 \mathrm{~A}$ & $-179.7(2)$ \\
\hline $\mathrm{C} 21 \mathrm{~B}-\mathrm{C} 22 \mathrm{~B}-\mathrm{C} 23 \mathrm{~B}-\mathrm{C} 18 \mathrm{~B}$ & $-0.7(4)$ & $\mathrm{C} 21 \mathrm{~A}-\mathrm{C} 22 \mathrm{~A}-\mathrm{C} 23 \mathrm{~A}-\mathrm{C} 18 \mathrm{~A}$ & $1.0(4)$ \\
\hline $\mathrm{C} 23 \mathrm{~B}-\mathrm{O} 2 \mathrm{~B}-\mathrm{B} 1 \mathrm{~B}-\mathrm{O} 1 \mathrm{~B}$ & $-1.1(3)$ & $\mathrm{C} 23 \mathrm{~A}-\mathrm{O} 2 \mathrm{~A}-\mathrm{B} 1 \mathrm{~A}-\mathrm{O} 1 \mathrm{~A}$ & $1.0(3)$ \\
\hline $\mathrm{C} 23 \mathrm{~B}-\mathrm{O} 2 \mathrm{~B}-\mathrm{B} 1 \mathrm{~B}-\mathrm{C} 11 \mathrm{~B}$ & $-178.7(2)$ & $\mathrm{C} 23 \mathrm{~A}-\mathrm{O} 2 \mathrm{~A}-\mathrm{B} 1 \mathrm{~A}-\mathrm{C} 11 \mathrm{~A}$ & $-177.8(2)$ \\
\hline $\mathrm{C} 23 \mathrm{~B}-\mathrm{C} 18 \mathrm{~B}-\mathrm{C} 19 \mathrm{~B}-\mathrm{C} 20 \mathrm{~B}$ & $0.7(4)$ & $\mathrm{C} 23 \mathrm{~A}-\mathrm{C} 18 \mathrm{~A}-\mathrm{C} 19 \mathrm{~A}-\mathrm{C} 20 \mathrm{~A}$ & $0.6(4)$ \\
\hline $\mathrm{B} 1 \mathrm{~B}-\mathrm{O} 1 \mathrm{~B}-\mathrm{C} 18 \mathrm{~B}-\mathrm{C} 19 \mathrm{~B}$ & $177.0(2)$ & $\mathrm{B} 1 \mathrm{~A}-\mathrm{O} 1 \mathrm{~A}-\mathrm{C} 18 \mathrm{~A}-\mathrm{C} 19 \mathrm{~A}$ & $-178.8(3)$ \\
\hline $\mathrm{B} 1 \mathrm{~B}-\mathrm{O} 1 \mathrm{~B}-\mathrm{C} 18 \mathrm{~B}-\mathrm{C} 23 \mathrm{~B}$ & $-0.6(3)$ & $\mathrm{B} 1 \mathrm{~A}-\mathrm{O} 1 \mathrm{~A}-\mathrm{C} 18 \mathrm{~A}-\mathrm{C} 23 \mathrm{~A}$ & $1.0(3)$ \\
\hline $\mathrm{B} 1 \mathrm{~B}-\mathrm{O} 2 \mathrm{~B}-\mathrm{C} 23 \mathrm{~B}-\mathrm{C} 18 \mathrm{~B}$ & $0.7(3)$ & $\mathrm{B} 1 \mathrm{~A}-\mathrm{O} 2 \mathrm{~A}-\mathrm{C} 23 \mathrm{~A}-\mathrm{C} 18 \mathrm{~A}$ & $-0.3(3)$ \\
\hline $\mathrm{B} 1 \mathrm{~B}-\mathrm{O} 2 \mathrm{~B}-\mathrm{C} 23 \mathrm{~B}-\mathrm{C} 22 \mathrm{~B}$ & $-177.1(2)$ & $\mathrm{B} 1 \mathrm{~A}-\mathrm{O} 2 \mathrm{~A}-\mathrm{C} 23 \mathrm{~A}-\mathrm{C} 22 \mathrm{~A}$ & $-179.7(3)$ \\
\hline $\mathrm{B} 1 \mathrm{~B}-\mathrm{C} 11 \mathrm{~B}-\mathrm{C} 12 \mathrm{~B}-\mathrm{C} 13 \mathrm{~B}$ & $124.1(3)$ & $\mathrm{B} 1 \mathrm{~A}-\mathrm{C} 11 \mathrm{~A}-\mathrm{C} 12 \mathrm{~A}-\mathrm{C} 13 \mathrm{~A}$ & $-49.1(3)$ \\
\hline $\mathrm{B} 1 \mathrm{~B}-\mathrm{C} 11 \mathrm{~B}-\mathrm{C} 12 \mathrm{~B}-\mathrm{C} 17 \mathrm{~B}$ & $-53.5(3)$ & $\mathrm{B} 1 \mathrm{~A}-\mathrm{C} 11 \mathrm{~A}-\mathrm{C} 12 \mathrm{~A}-\mathrm{C} 17 \mathrm{~A}$ & $129.4(2)$ \\
\hline
\end{tabular}

